\title{
Reactivity of Styrenes in Micelles: Safe, Selective, and Sustainable Functionalization with Azides and Carboxylic Acids
}

Susanta Hazra, ${ }^{\dagger}$ Gaganpreet Kaur, ${ }^{\dagger}$ Sachin Handa ${ }^{\dagger}, *$

$\dagger 2320$ S. Brook St., Department of Chemistry, University of Louisville, Louisville, Kentucky 40292, United States

*Correspondence Author: sachin.handa@louisville.edu

Supporting information includes:

Total number of Pages: 43

Total number of Tables: 6

Total number of Schemes: 6

1. General experimental details $\quad \mathrm{S} 2$

2. Reaction optimization (Table S1-S5) S3-S6

3. General procedure for oxyazidation of styrenes $\quad$ S7

4. General procedure for oxyacyoxylation of styrenes $\quad$ S8

5. General substrate scope (Table S6) S9

6. General procedure for one-pot reduction of azidoketones to azidoalcohols S10

7. General procedure for synthesis of $\beta$-keto triazole in one-pot S10-S11

8. Gram scale reaction S11-S12

9. Procedure for control experiments $\quad \mathrm{S} 12$

$\begin{array}{ll}\text { 10. Analytical data } & \text { S13-S22 }\end{array}$

$\begin{array}{ll}\text { 11. References } & \text { S23 }\end{array}$

12. ${ }^{1} \mathrm{H} \&{ }^{13} \mathrm{C}$ spectra of compounds 2-30 S24-S43 


\section{GENERAL EXPERIMENTAL DETAILS}

Unless otherwise stated, all reactions were performed under argon atmosphere. All NMR spectra were recorded at $25^{\circ} \mathrm{C}$ on Varian Unity INOVA (400 and $500 \mathrm{MHz}$ ) spectrometers. Reported chemical shifts are referenced to residual solvent peaks. ${ }^{1}$ All chemical shifts $(\delta)$ are reported in ppm and coupling constants $(J)$ in Hz. GCMS data was obtained using a Thermo Scientific Trace 1300 Gas Chromatograph coupled with a Thermo Scientific ISQ-QD Single Quadrupole Mass Spectrometer. HRMS spectra were obtained on a Thermo Electron MAT 95XP mass spectrometer using either electron ionization (EI) or chemical ionization (CI). TLC plates (UV 254 indicator, glass backed, thickness $200 \mathrm{~mm}$ ) and silica gel (standard grade, 230 -400 mesh) were purchased from Merck. Pure NMR solvents were purchased from Cambridge Isotopes Laboratories. Styrenes, NBS, NCS, NIS, BHT, TEMPO-free radical were purchased from commercial sources such as Sigma Aldrich, Alfa-Asear, Acros Organics, Combi-Blocks, and Oakwood chemicals. The reactions were performed in $4 \mathrm{~mL}$ close-cap microwave vials, and large-scale reactions in $50 \mathrm{~mL}$ round-bottom flasks. Microwave vials were purchased from VWR International and Biotage. Reaction vials were also recycled and reused. Bulk aqueous solution of surfactant PS-750-M was prepared in HPLC grade water ${ }^{2}$ and thoroughly purged with argon before use. Melting points were determined using a MEL-TEMP II melting point apparatus with samples in Kimble Kimex 51 capillaries (1.5-1.8 x 90 mm). 


\section{REACTION OPTIMIZATION}

Table S1. Effect of temperature
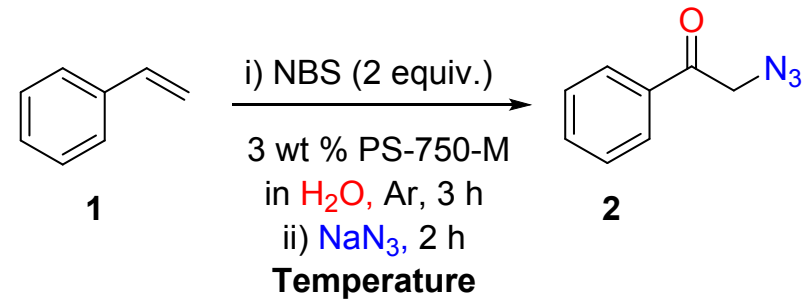

Temperature

\begin{tabular}{|c|c|c|}
\hline Entry & Temperature $\left({ }^{\circ} \mathrm{C}\right)$ & $2(\%)^{a}$ \\
\hline 1 & $\mathrm{rt}$ & traces $(35)^{b}$ \\
\hline 2 & $35^{\circ} \mathrm{C}$ & 25 \\
\hline 3 & $45^{\circ} \mathrm{C}$ & 55 \\
\hline 4 & $60^{\circ} \mathrm{C}$ & 76 \\
\hline 5 & $60{ }^{\circ} \mathrm{C}$ & $22^{c}$ \\
\hline 6 & $\begin{array}{c}20 \mathrm{~mol} \% \mathrm{Bu}_{4} \mathrm{NCl} \text { additive, } 45^{\circ} \mathrm{C} \\
\text { after azide addition }\end{array}$ & $69^{d}$ \\
\hline 7 & $\begin{array}{c}20 \mathrm{~mol} \% \mathrm{Bu}_{4} \mathrm{NCl} \text { additive, } \mathrm{rt} \text { after } \\
\text { azide addition }\end{array}$ & $52^{d}$ \\
\hline
\end{tabular}

Conditions: 1 (0.5 mmol), NBS (1 mmol, 2 equiv.), $1 \mathrm{~mL} 3 \mathrm{wt} \%$ PS-750-M in $\mathrm{H}_{2} \mathrm{O}(0.5 \mathrm{M})$, $60{ }^{\circ} \mathrm{C}, 3 \mathrm{~h}$ under argon atmosphere. Afterwards, $\mathrm{NaN}_{3}$ (0.75 mmol, 1.5 equiv.), $60{ }^{\circ} \mathrm{C}, 2 \mathrm{~h}$. ${ }^{a}$ Isolated yields. ${ }^{b}$ Reaction was carried out for $24 \mathrm{~h}$. ${ }^{c}$ Reaction was conducted under oxygen atmosphere. ${ }^{d}$ Bromination step was performed at $60{ }^{\circ} \mathrm{C}$ for $3 \mathrm{~h}$ and azidation using $20 \mathrm{~mol} \%$ $\mathrm{Bu}_{4} \mathrm{NCl}$ additive and 1.5 equiv $\mathrm{NaN}_{3}, 2 \mathrm{~h}$. 
Table S2. Effect of halogenating agents

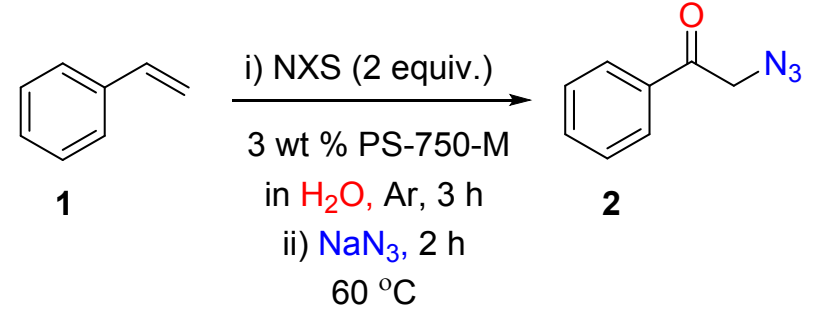

\begin{tabular}{ccc}
\hline Entry & NXS (2 eq. $)$ & $\mathbf{2}(\%)^{a}$ \\
\hline $\mathbf{1}$ & NBS & $\mathbf{7 6}$ \\
2 & NCS & traces \\
3 & NIS & $36^{b}$
\end{tabular}

Conditions: 1 (0.5 mmol), NXS (1 mmol, 2 equiv.), $1 \mathrm{~mL} 3$ wt \% PS-750-M in $\mathrm{H}_{2} \mathrm{O}(0.5 \mathrm{M})$, $60{ }^{\circ} \mathrm{C}, 3 \mathrm{~h}$ under argon atmosphere. Afterwards, $\mathrm{NaN}_{3}$ (0.75 mmol, 1.5 equiv.), $60{ }^{\circ} \mathrm{C}, 2 \mathrm{~h}$. ${ }^{a}$ Isolated yields. ${ }^{b}$ In this case, $35 \%$ (1, 2-diiodoethyl)benzene was also isolated in addition to $36 \% 2$.

Table S3. Effect of stoichiometry of NBS

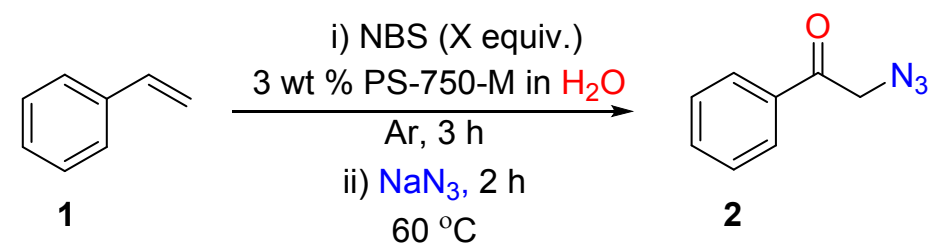

\begin{tabular}{ccc}
\hline entry & NBS (equiv.) & $\mathbf{2}(\%)^{a}$ \\
\hline 1 & none & 0 \\
2 & 1.0 & 31 \\
3 & 1.5 & 55 \\
4 & 2.0 & 76 \\
$\mathbf{5}$ & $\mathbf{2 . 5}$ & $\mathbf{8 5}$ \\
6 & 3.0 & 72
\end{tabular}

Conditions: all the reactions were conducted using $0.5 \mathrm{mmol}$ styrene, $0.75 \mathrm{mmol}_{\text {of } \mathrm{NaN}_{3} \text {, and }}$ $1.0 \mathrm{~mL} 3 \mathrm{wt} \%$ PS-750-M in $\mathrm{H}_{2} \mathrm{O}$ at $60{ }^{\circ} \mathrm{C}$ for $5 \mathrm{~h}$ (overall time) under argon atmosphere. ${ }^{a}$ Isolated yields.

Table S4. Effect of solvents

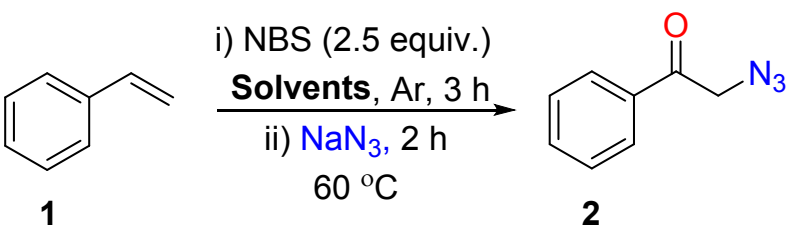




\begin{tabular}{ccc}
\hline entry & reaction solvent & $\mathbf{2}(\%)^{a}$ \\
\hline 1 & neat water & 65 \\
2 & $\mathrm{CH}_{3} \mathrm{CN}$ & 42 \\
3 & $1,4-$ dioxane & 82 \\
4 & $3 \mathrm{wt} \%$ aq. PS-750-M, $1 \mathrm{~mL}$ & 85 \\
6 & $3 \mathrm{wt} \%$ aq. PS-750-M, $0.5 \mathrm{~mL}^{b}$ & 76 \\
6 & $3 \mathrm{wt} \%$ aq. PS-750-M, $2 \mathrm{~mL}^{c}$ & 58 \\
\hline
\end{tabular}

Conditions: all the reactions were carried using $0.5 \mathrm{mmol}$ styrene, NBS (1.25 mmol), 0.75 mmol of $\mathrm{NaN}_{3}$ and $1 \mathrm{~mL}, 0.5(\mathrm{M})$ global concentration) of solvent at $60{ }^{\circ} \mathrm{C}$ for $5 \mathrm{~h}$ (overall time) under argon atmosphere. ${ }^{a}$ Isolated yields. ${ }^{b}$ Using 1 (M) global concentration. ${ }^{c}$ Using $0.25(\mathrm{M})$ global concentration. 
Table S5. The nature of nucleophiles

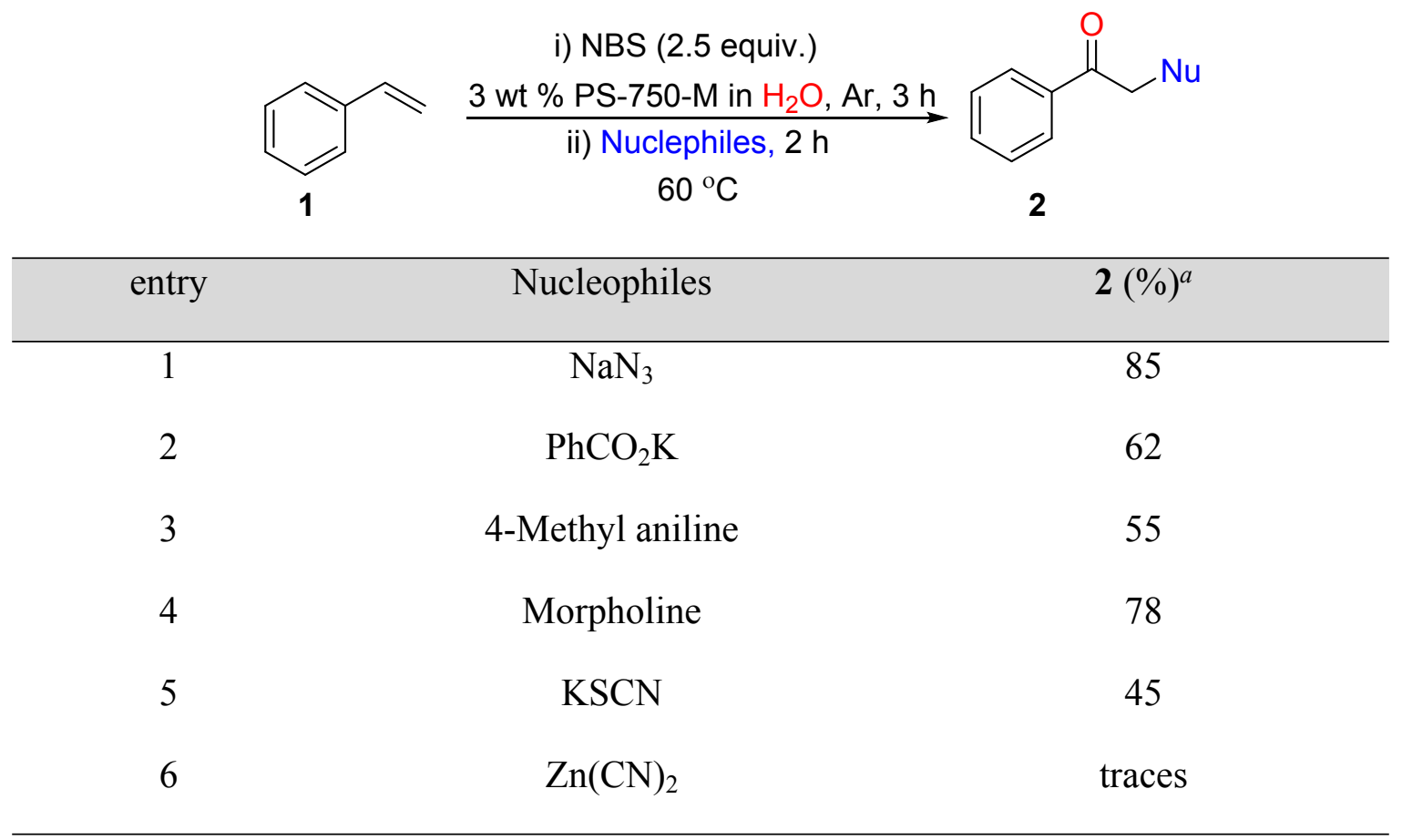

Conditions: all the reactions were carried using $0.5 \mathrm{mmol}$ styrene, NBS (1.25 mmol), 0.75 mmol of respective nucleophile, and $1 \mathrm{~mL} 3 \mathrm{wt} \% \mathrm{PS}-750-\mathrm{M}$ in $\mathrm{H}_{2} \mathrm{O}$ at $60{ }^{\circ} \mathrm{C}$ for $5 \mathrm{~h}$ under argon atmosphere. ${ }^{a}$ Isolated yields. 


\section{GENERAL PROCEDURE FOR OXYAZIDATION OF STYRENES}

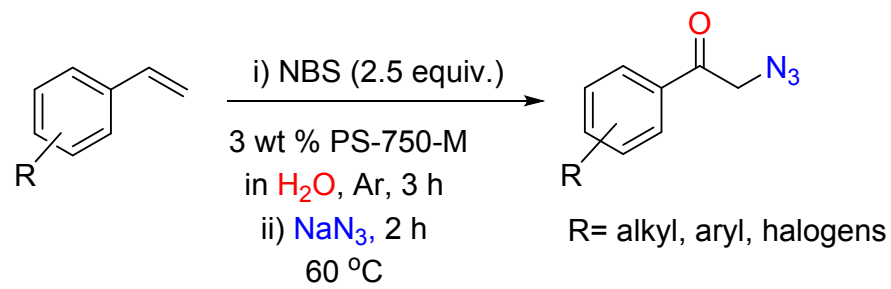

Scheme S1. One-pot oxyazidation.

A $4 \mathrm{~mL}$ oven-dried reaction vial was charged with a PTFE-coated stirrer bar and $1.25 \mathrm{mmol}$ NBS (2.5 equiv.). After closing with a rubber septum, the vial was evacuated and backfilled with argon. Afterwards, $0.5 \mathrm{mmol}$ styrene (1 equiv.) and $1 \mathrm{~mL} 3 \mathrm{wt} \%$ aqueous PS-750-M were added to the reaction mixture (see Scheme S1). Septum was wrapped with PTFE tape and a parafilm. The reaction mixture was heated at $60{ }^{\circ} \mathrm{C}$ on a pre-heated oil bath. The reaction progress was monitored by TLC. After complete consumption of styrene (generally $1^{\text {st }}$ step took 3-13 h depending on the substrate), reaction was allowed to come to $\mathrm{rt}$, and $0.75 \mathrm{mmol}$ $\mathrm{NaN}_{3}$ (1.5 equiv.) was added to the reaction mixture. The mixture was stirred for additional 2 $\mathrm{h}$ (for most cases the $2^{\text {nd }}$ step was completed within $2 \mathrm{~h}$ ). After reaction completion, reaction was allowed to come to rt again. The septum was removed and $1.0 \mathrm{~mL}$ EtOAc was added to the reaction mixture, which was then stirred for a minute at rt. The organic layer was removed with the use of a pipette. This extraction process was repeated for an additional time. The combined organic layers were dried over anhydrous sodium sulfate. Volatiles were removed under reduced pressure to obtain crude product, which was further purified by flash chromatography over silica gel using EtOAc/hexanes as eluent. The isolated products were characterized by various spectroscopic techniques and the experimental data matched with literature for known compounds. ${ }^{3-5}$ Compounds $\mathbf{2 , 3}, \mathbf{4}, \mathbf{5}, \mathbf{7 ,} \mathbf{8}, \mathbf{9}, 11$ are known in literature, see the substrate scope table in section 5. 


\section{GENERAL PROCEDURE FOR OXYACYLOXYLATION OF STYRENES}

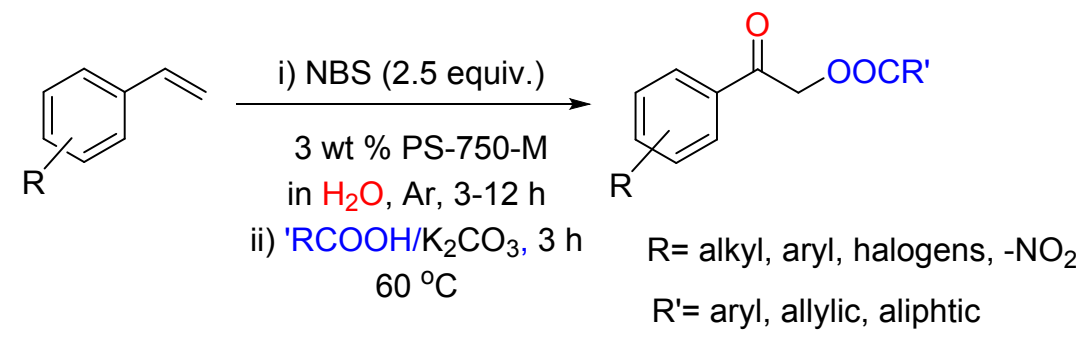

Scheme S2. One-pot oxyacyloxylation.

A $4 \mathrm{~mL}$ oven-dried reaction vial containing PTFE-coated stirrer bar, $1.25 \mathrm{mmol}$ NBS (2.5 equiv.) was added. The reaction vial was closed with a rubber septum and vial was evacuated and backfilled with argon. Afterwards, $0.5 \mathrm{mmol}$ styrene (1 equiv.) and $1 \mathrm{~mL} 3 \mathrm{wt} \%$ aqueous PS-750-M were added to the reaction mixture (see Scheme S2). Septum was wrapped with PTFE tape and a parafilm. The reaction mixture was heated at $60{ }^{\circ} \mathrm{C}$ on a pre-heated oil bath. The reaction progress was monitored by TLC. After complete consumption of styrene (generally $1^{\text {st }}$ step took 3-12 h depending on the substrate), mixture was allowed to come to rt. Then, $0.75 \mathrm{mmol}$ carboxylic acid (1.5 equiv.) and $0.75 \mathrm{mmol} \mathrm{K}_{2} \mathrm{CO}_{3}$ were added to the reaction mixture. Reaction mixture was stirred for additional $3 \mathrm{~h}$ (for most cases, the $2^{\text {nd }}$ step was completed within $3 \mathrm{~h}$. See Table S6 on next page for reaction time. After reaction completion, mixture was allowed to come to rt. The septum was removed and $1.0 \mathrm{~mL}$ EtOAc was added to the reaction mixture, which was then stirred for a minute at rt. An organic layer was removed with the use of a pipette. This extraction process was repeated for an additional time. Combined organic layers were dried over anhydrous sodium sulfate. Volatiles were removed under reduced pressure to obtain the crude product, which was further purified by flash chromatography over silica gel using EtOAc/hexanes as eluent. The isolated products were characterized by various spectroscopic techniques and the experimental data matched with literature for known compounds. ${ }^{6}$ Compounds 12, 13, 14, 16, 17, 19, 20, 23 are known in literature, see the substrate scope table given in sec 5 . 


\section{GENERAL SUBSTRATE SCOPE}

Table S6. Scope study

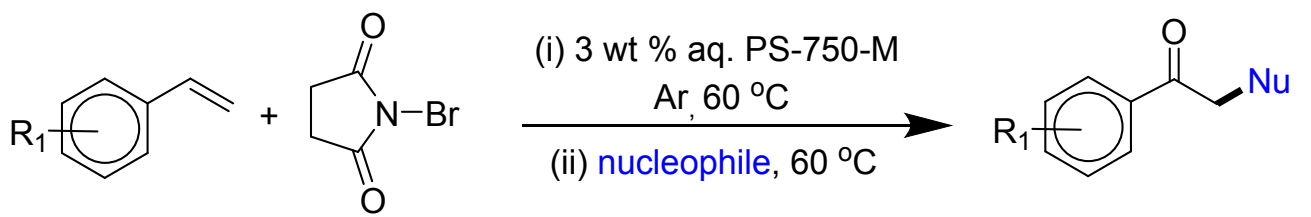
nucelophile $=$ azide, carboxylic acid, thiocyanate, amine<smiles>Cc1ccc(C(=O)CN)cc1</smiles><smiles>NCC(=O)c1ccc(-c2cccc3ccccc23)cc1</smiles><smiles>CC(N)C(=O)c1ccc(Cl)cc1</smiles><smiles>NCC(=O)c1ccc(F)c(Br)c1</smiles><smiles>NCC(=O)c1ccc(Cl)cc1</smiles><smiles>N#CCC(=O)c1ccccc1</smiles><smiles>Cc1ccc(C(=O)COC(=O)c2ccccc2)cc1</smiles>

$1057 \%(12 \mathrm{~h})$

$1163 \%(8 \mathrm{~h})$

$1245 \%(5 \mathrm{~h})$

$1365 \%(6 \mathrm{~h})$<smiles>O=C(COC(=O)c1ccccc1)c1ccccc1</smiles><smiles>CC(C)(C)c1ccc(C(=O)COC(=O)c2ccccc2)cc1</smiles>

$1560 \%(5 \mathrm{~h})$<smiles>O=C(COC(=O)c1ccccc1)c1ccc(F)cc1</smiles><smiles>O=C(COC(=O)c1ccc(Br)cc1)c1ccccc1</smiles>

17 58\% (8 h)<smiles>O=C(COC(=O)c1ccccc1)c1ccc(F)c(Br)c1</smiles>

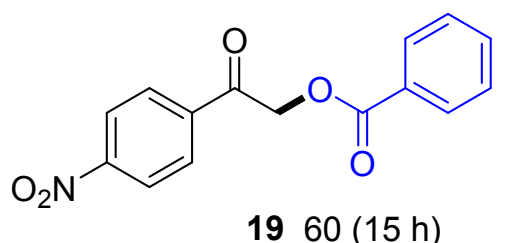<smiles>O=C(Cc1ccccc1)OCC(=O)c1ccccc1</smiles>

$2365 \%(6 h)$<smiles>COc1ccc(NCC(=O)c2ccccc2)cc1</smiles>

$2455 \%(5 \mathrm{~h})$<smiles>CC(=O)OCc1ccc(C(=O)COC(=O)C2=CCCCC2)cc1</smiles>

22 64\% (6 h)

25 78\% (6 h) 


\section{GENERAL PROCEDURE FOR ONE-POT REDUCTION OF AZIDOKETONES TO AZIDO ALCOHOLS}

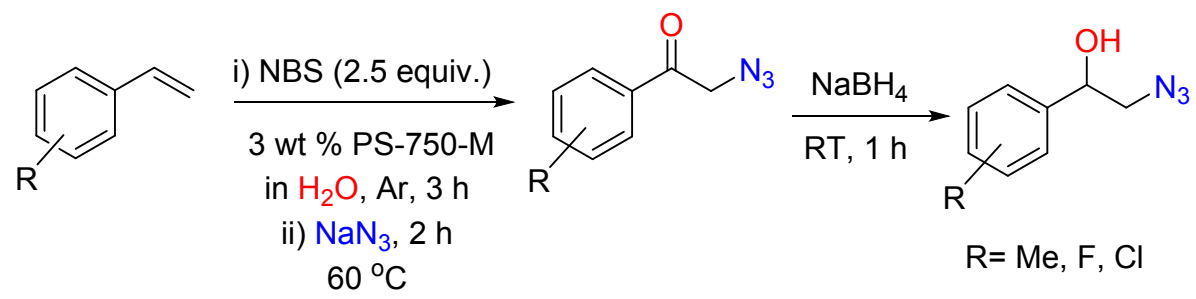

Scheme S3. One-pot reduction.

After following the general procedure described in section 3 on page S7, reaction mixture was allowed to cool to rt. Then, $0.6 \mathrm{mmol} \mathrm{NaBH}_{4}$ (1.2 equiv.) was added to the reaction mixture and mixture was stirred at $\mathrm{rt}$ for $1 \mathrm{~h}$ (see Scheme S3). After complete carbonyl reduction as monitored by TLC, septum was removed, and $1.0 \mathrm{~mL}$ EtOAc was added to the reaction mixture. The reaction mixture was stirred for a minute at $\mathrm{rt}$. An organic layer was removed with the use of a pipette. This extraction process was repeated for an additional time. The combined organic layers were dried over anhydrous sodium sulfate. Volatiles were removed under reduced pressure to obtain the crude product, which was further purified by flash chromatography over silica gel using EtOAc/hexanes as eluent (1:4).

\section{GENERAL PROCEDURE FOR SYNTHESIS OF $\beta$-KETO TRIAZOLES IN ONE POT}

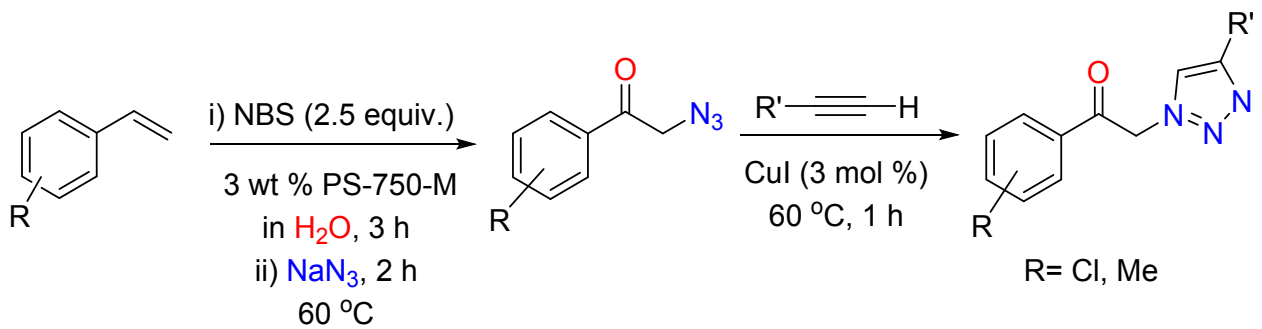

Scheme S4. One-pot triazole formation.

After following the general procedure mentioned in section 3, the reaction mixture was cooled down to rt. Then, $0.5 \mathrm{mmol}$ alkynes ( 1 equiv.) and $0.015 \mathrm{mmol}(3 \mathrm{~mol} \%) \mathrm{CuI}$ were added to the reaction mixture, and mixture was stirred at $60{ }^{\circ} \mathrm{C}$ (see Scheme S4). After $1 \mathrm{~h}$, the reaction 
mixture was cooled down to rt. $2 \mathrm{~mL}$ ice cooled water was added to it. During addition of water, crude product were precipitated out from the solution. The precipitate was filtered off and crude product was purified by flash chromatography over silica gel using EtOAc/hexanes as eluent to obtain the desired $\beta$-keto triazoles.

\section{GRAM SCALE REACTION}

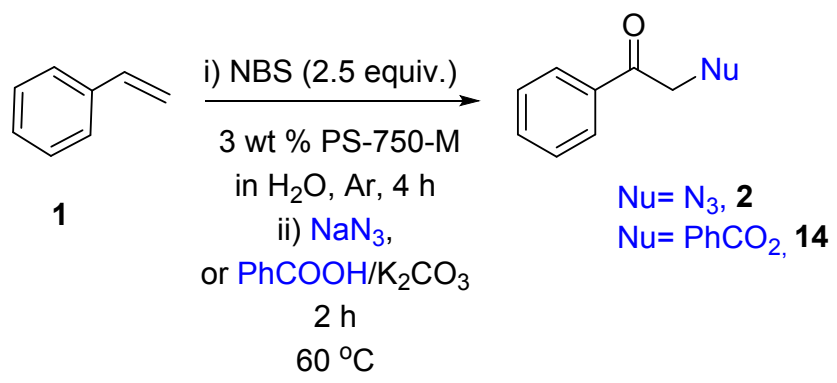

Scheme S5. Gram-scale reactions.

A $100 \mathrm{~mL}$ oven-dried round bottom flask was charged with a PTFE-coated stirrer bar and 23.8 mmol of NBS (2.5 equiv.). After closing with a rubber septum, the round bottom flask was evacuated and backfilled with argon balloon. Afterwards, 9.5 mmol styrene $(1 \mathrm{~g})$ and $19 \mathrm{~mL} 3$ wt $\%$ aqueous PS-750-M was added to the reaction mixture (see Scheme S5). Septum was wrapped with PTFE tape and parafilm. The reaction mixture was heated at $60{ }^{\circ} \mathrm{C}$ on a preheated oil bath. The reaction was monitored by TLC. After consumption of styrene (after $4 \mathrm{~h}$ ), reaction mixture was cooled down to rt, and $14.2 \mathrm{mmol} \mathrm{NaN} \mathrm{N}_{3}$ (1.5 equiv.) or $\mathrm{PhCOOH} / \mathrm{K}_{2} \mathrm{CO}_{3}$ $(1: 1)$ was added to the reaction mixture, and mixture was stirred for additional $2 \mathrm{~h}$. After reaction completion, reaction mixture was allowed to cool to rt. A septum was removed and 10 $\mathrm{mL}$ EtOAc was added to the reaction mixture, which was then stirred for a minute at $\mathrm{rt}$. The organic layer was removed with the help of a pipette. This extraction process was repeated for an additional time. The combined organic layers were dried over anhydrous sodium sulfate. Volatiles were removed under reduced pressure to obtain crude product, which was further purified by flash chromatography over silica gel using EtOAc/hexanes as eluent (1:9). 85\% 
yield (1.3 g) of 2-azido-1-phenylethan-1-one 2 and 60\% yield (1.4 g) of 2-oxo-2-phenylethyl benzoate 14 was obtained.

\section{PROCEDURE FOR CONTROL EXPERIMENTS}

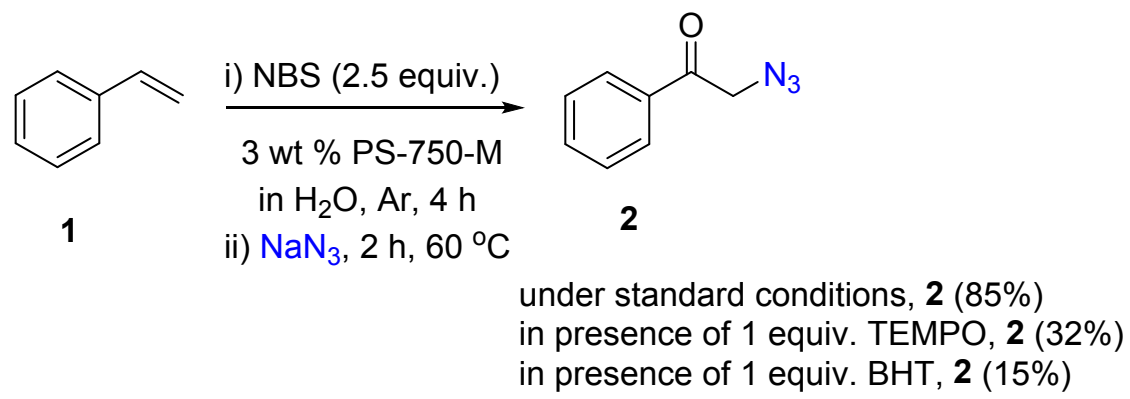

Scheme S6. Control experiments.

In a $4 \mathrm{~mL}$ oven-dried reaction vial containing PTFE-coated stirrer bar, $1.25 \mathrm{mmol}$ NBS (2.5 equiv.) and $0.5 \mathrm{mmol}$ of radical scavenger (TEMPO or BHT, 1 equiv.) were added. The reaction vial was closed with a rubber septum. The vial was evacuated and backfilled with argon. Afterwards, $0.5 \mathrm{mmol}$ styrene (1 equiv.) and $1 \mathrm{~mL} 3 \mathrm{wt} \%$ aqueous PS-750-M were added to the reaction mixture (see Scheme S6). Septum was wrapped with PTFE tape and parafilm. A reaction mixture was heated at $60{ }^{\circ} \mathrm{C}$ on a pre-heated oil bath for $3 \mathrm{~h}$. The mixture was allowed to come to rt after $3 \mathrm{~h}$ and $0.75 \mathrm{mmol} \mathrm{NaN}_{3}$ (1.5 equiv.) was added to it. The mixture was stirred for additional $2 \mathrm{~h}$ at $60{ }^{\circ} \mathrm{C}$. Afterwards, reaction mixture was allowed to cool to $\mathrm{rt}$. Septum was removed and $1.0 \mathrm{~mL}$ EtOAc was added to the reaction mixture, which was then stirred for a minute at rt. An organic layer was removed with the use of a pipette. This extraction process was repeated for an additional time. The combined organic layers were dried over anhydrous sodium sulfate. Volatiles were removed under reduced pressure to obtain crude product, which was further purified by flash chromatography over silica gel using 5\% EtOAc and hexane as eluent. The reaction with BHT was also analyzed by GC-MS after $1 \mathrm{~h}$ with adding $\mathrm{NaN}_{3}$. The GC-MS analysis of the reaction mixture suggested the formation of radical intermediates for this transformation. 


\section{ANALYTICAL DATA}

2-Azido-1-phenylethan-1-one (2)<smiles>NCC(=O)c1ccccc1</smiles>

Yellow liquid, yield $68 \mathrm{mg}(85 \%), \mathrm{R}_{f} 0.55$ (1:9 EtOAc:hexanes). ${ }^{1} \mathrm{H}$ NMR (500 MHz, $\left.\mathrm{CDCl}_{3}\right)$ : $\delta 7.90(\mathrm{~d}, J=10 \mathrm{~Hz}, 2 \mathrm{H}), 7.64-7.61(\mathrm{~m}, 1 \mathrm{H}), 7.51-7.48(\mathrm{~m}, 2 \mathrm{H}), 4.56(\mathrm{~s}, 2 \mathrm{H})$.

\section{2-Azido-1-(p-tolyl)ethan-1-one (3)}<smiles>Cc1ccc(C(=O)CN)cc1</smiles>

White solid, yield $72 \mathrm{mg}(82 \%), \mathrm{R}_{f} 0.56$ (1:9 EtOAc:hexanes). ${ }^{1} \mathrm{H}$ NMR (400 $\mathrm{MHz}, \mathrm{CDCl}_{3}$ ): $\delta 7.80(\mathrm{~d}, J=8 \mathrm{~Hz}, 2 \mathrm{H}), 7.29(\mathrm{~d}, J=8 \mathrm{~Hz}, 2 \mathrm{H}), 4.53(\mathrm{~s}, 2 \mathrm{H}), 2.43(\mathrm{~s}, 3 \mathrm{H})$.

\section{2-Azido-1-(4-(tert-butyl)phenyl)ethan-1-one (4)}

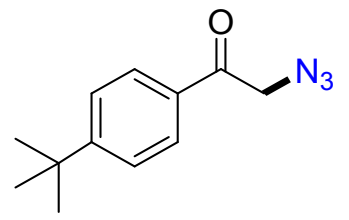

Colorless liquid, yield $67 \mathrm{mg}(62 \%), \mathrm{R}_{f} 0.61$ (1:9 EtOAc:hexanes). ${ }^{1} \mathrm{H}$ NMR (400 MHz, $\left.\mathrm{CDCl}_{3}\right): \delta 7.94(\mathrm{~d}, J=8 \mathrm{~Hz}, 2 \mathrm{H}), 7.51(\mathrm{~d}, J=8 \mathrm{~Hz}, 2 \mathrm{H}), 4.44(\mathrm{~s}, 2 \mathrm{H}), 1.36(\mathrm{~s}, 9 \mathrm{H})$. 


\section{1-([1,1'-biphenyl]-4-yl)-2-azidoethan-1-one (5)}<smiles>NCC(=O)c1ccc(-c2ccccc2)cc1</smiles>

White solid, yield $77 \mathrm{mg}(65 \%), \mathrm{R}_{f} 0.43$ (1:9 EtOAc:hexanes). ${ }^{1} \mathrm{H}$ NMR (400 MHz, $\mathrm{CDCl}_{3}$ ): $\delta 7.99(\mathrm{~d}, J=8 \mathrm{~Hz}, 2 \mathrm{H}), 7.72(\mathrm{~d}, J=8 \mathrm{~Hz}, 2 \mathrm{H}), 7.63(\mathrm{~d}, J=8 \mathrm{~Hz}, 2 \mathrm{H}), 7.49-7.42(\mathrm{~m}, 3 \mathrm{H})$, $4.60(\mathrm{~s}, 2 \mathrm{H})$.

\section{2-Azido-1-(4-(naphthalen-1-yl)phenyl)ethan-1-one (6)}<smiles>NCC(=O)c1ccc(-c2cccc3ccccc23)cc1</smiles>

White solid, yield $74 \mathrm{mg}(52 \%), \mathrm{mp}=110-112^{\circ} \mathrm{C}, \mathrm{R}_{f} 0.50$ (1:9 EtOAc:hexanes). ${ }^{1} \mathrm{H}$ NMR (400 $\left.\mathrm{MHz}, \mathrm{CDCl}_{3}\right): \delta 8.34(\mathrm{~d}, J=8 \mathrm{~Hz}, 1 \mathrm{H}), 8.03(\mathrm{~d}, J=8 \mathrm{~Hz}, 2 \mathrm{H}), 7.85(\mathrm{~d}, J=8 \mathrm{~Hz}, 1 \mathrm{H}), 7.77$ $(\mathrm{d}, J=8 \mathrm{~Hz}, 1 \mathrm{H}), 7.63-7.58(\mathrm{~m}, 3 \mathrm{H}), 7.50-7.49(\mathrm{~m}, 1 \mathrm{H}), 7.25(\mathrm{~d}, J=8 \mathrm{~Hz}, 2 \mathrm{H}), 4.62(\mathrm{~s}, 2 \mathrm{H})$. ${ }^{13} \mathrm{C}$ NMR (100 MHz, $\left.\mathrm{CDCl}_{3}\right): \delta$ 192.3, 134.7, 133.0, 132.3, 132.1, 130.0, 129.5, 129.4, 128.4, 128.0, 127.4, 127.2, 126.8, 126.2, 122.9, 54.8. HRMS (EI) m/z: $\left[\mathrm{M}-\mathrm{N}_{2}+\mathrm{H}\right]^{+}$Calcd for $\mathrm{C}_{18} \mathrm{H}_{13} \mathrm{ON} 259.0992$; found 259.0991 .

\section{2-Azido-1-(4-fluorophenyl)ethan-1-one (7) ${ }^{4,5}$}<smiles>NCC(=O)c1ccc(F)cc1</smiles>

White solid, yield $60 \mathrm{mg}(67 \%), \mathrm{R}_{f} 0.50$ (1:9 EtOAc:hexanes). ${ }^{1} \mathrm{H} \mathrm{NMR}\left(400 \mathrm{MHz}, \mathrm{CDCl}_{3}\right.$ ): $\delta$ 7.93-7.96 (m, 2H), $7.17(\mathrm{t}, J=8 \mathrm{~Hz}, 2 \mathrm{H}), 4.53(\mathrm{~s}, 2 \mathrm{H})$. 
<smiles>NCC(=O)c1ccc(Br)cc1</smiles>

White solid, yield $74 \mathrm{mg}(62 \%), \mathrm{R}_{f} 0.58$ (1:9 EtOAc:hexanes). ${ }^{1} \mathrm{H}$ NMR (400 $\mathrm{MHz}, \mathrm{CDCl}_{3}$ ): $\delta 7.85(\mathrm{~d}, J=12 \mathrm{~Hz}, 2 \mathrm{H}), 7.64(\mathrm{~d}, J=8 \mathrm{~Hz}, 2 \mathrm{H}), 4.40(\mathrm{~s}, 2 \mathrm{H})$.

\section{2-Azido-1-(4-chlorophenyl)propan-1-one (9) ${ }^{5}$}<smiles>CC(N)C(=O)c1ccc(Cl)cc1</smiles>

Reaction was performed with $E$-isomer of starting material. Colorless liquid, yield $68 \mathrm{mg}$ (65\%), $\mathrm{R}_{f} 0.51$ (1:9 EtOAc:hexanes). ${ }^{1} \mathrm{H}$ NMR (400 MHz, $\left.\mathrm{CDCl}_{3}\right): \delta 7.89(\mathrm{~d}, J=12 \mathrm{~Hz}, 2 \mathrm{H})$, $7.48(\mathrm{~d}, J=8 \mathrm{~Hz}, 2 \mathrm{H}), 4.64(\mathrm{q}, J=7.2 \mathrm{~Hz}, 1 \mathrm{H}), 1.57(\mathrm{~d}, J=8 \mathrm{~Hz}, 3 \mathrm{H})$.

\section{2-Azido-1-(3-bromo-4-fluorophenyl)ethan-1-one (10)}<smiles>NCC(=O)c1ccc(F)c(Br)c1</smiles>

Red oil, yield $74 \mathrm{mg}$ (57\%), $\mathrm{R}_{f} 0.50$ (1:9 EtOAc:hexanes). ${ }^{1} \mathrm{H}$ NMR (400 MHz, $\left.\mathrm{CDCl}_{3}\right): \delta 8.12$ $(\mathrm{dd}, J=8,4 \mathrm{~Hz}, 1 \mathrm{H}), 7.82-7.86(\mathrm{~m}, 1 \mathrm{H}), 7.21-7.24(\mathrm{~m}, 1 \mathrm{H}), 4.49(\mathrm{~s}, 2 \mathrm{H}) ;{ }^{13} \mathrm{C}$ NMR $(100 \mathrm{MHz}$ $\left.\mathrm{CDCl}_{3}\right): \delta 190.8,162.5(\mathrm{~d}, J=256 \mathrm{~Hz}), 134.1,131.9,129.3(\mathrm{~d}, J=33.2 \mathrm{~Hz}), 117.2(\mathrm{~d}, J=91.2$ Hz), $110.4\left(\mathrm{~d}, J=22 \mathrm{~Hz}\right.$ ), 54.9. HRMS (EI) m/z: $\left[\mathrm{M}-\mathrm{N}_{2}+\mathrm{H}\right]^{+}$Calcd for $\mathrm{C}_{8} \mathrm{H}_{6} \mathrm{ONFBr}$ 229.9611; found 229.9612. 
<smiles>NCC(=O)c1ccc(Cl)cc1</smiles>

White solid, yield $62 \mathrm{mg}(63 \%), \mathrm{R}_{f} 0.52$ (1:9 EtOAc:hexanes). ${ }^{1} \mathrm{H}$ NMR (400 $\mathrm{MHz}, \mathrm{CDCl}_{3}$ ): $\delta 7.78(\mathrm{~d}, J=8 \mathrm{~Hz}, 2 \mathrm{H}), 7.65(\mathrm{~d}, J=8 \mathrm{~Hz}, 2 \mathrm{H}), 4.52(\mathrm{~s}, 2 \mathrm{H})$.

\section{1-Phenyl-2-thiocyanatoethan-1-one (12) ${ }^{8}$}<smiles>N#CCC(=O)c1ccccc1</smiles>

White solid, yield $40 \mathrm{mg}(45 \%), \mathrm{R}_{f} 0.30$ (1:4 EtOAc:hexanes). ${ }^{1} \mathrm{H}$ NMR (400 $\mathrm{MHz}, \mathrm{CDCl}_{3}$ ): $\delta 7.92(\mathrm{~d}, J=4 \mathrm{~Hz}, 2 \mathrm{H}), 7.66(\mathrm{~m}, 1 \mathrm{H}), 7.52(\mathrm{~d}, J=4 \mathrm{~Hz}, 2 \mathrm{H}), 4.73(\mathrm{~s}, 2 \mathrm{H})$.

\section{2-Oxo-2-(p-tolyl)ethyl benzoate (13) ${ }^{6}$}<smiles>Cc1ccc(C(=O)COC(=O)c2ccccc2)cc1</smiles>

White solid, yield $83 \mathrm{mg},(65 \%), \mathrm{R}_{f} 0.51$ (1:9 EtOAc:hexanes). ${ }^{1} \mathrm{H}$ NMR (400 MHz, $\mathrm{CDCl}_{3}$ ): $\delta 8.14(\mathrm{~d}, J=12 \mathrm{~Hz}, 2 \mathrm{H}), 7.87(\mathrm{~d}, J=8 \mathrm{~Hz}, 2 \mathrm{H}), 7.59$ (t, $J=12 \mathrm{~Hz}, 1 \mathrm{H}), 7.46(\mathrm{t}, J=12 \mathrm{~Hz}$, 2H), $7.30(\mathrm{~d}, J=8 \mathrm{~Hz}, 2 \mathrm{H}), 5.56(\mathrm{~s}, 2 \mathrm{H}), 2.43(\mathrm{~s}, 3 \mathrm{H})$.

\section{2-Oxo-2-phenylethyl benzoate (14)}<smiles>O=C(COC(=O)c1ccccc1)c1ccccc1</smiles> 
White solid, yield $74 \mathrm{mg}$ (62\%), $\mathrm{R}_{f} 0.50$ (1:9 EtOAc:hexanes). ${ }^{1} \mathrm{H}$ NMR (400 $\mathrm{MHz}, \mathrm{CDCl}_{3}$ ): $\delta 8.15(\mathrm{~d}, J=8 \mathrm{~Hz}, 2 \mathrm{H}), 7.98(\mathrm{~d}, J=8 \mathrm{~Hz}, 2 \mathrm{H}), 7.58-7.65(\mathrm{~m}, 2 \mathrm{H}), 7.46-7.53(\mathrm{~m}, 4 \mathrm{H}), 5.59$ $(\mathrm{s}, 2 \mathrm{H})$.

2-(4-(tert-butyl)phenyl)-2-oxoethyl benzoate (15)<smiles>CC(C)(C)c1ccc(C(=O)COC(=O)c2ccccc2)cc1</smiles>

White solid, yield $89 \mathrm{mg}(60 \%), \mathrm{mp}=105-107^{\circ} \mathrm{C}, \mathrm{R}_{f} 0.60$ (1:9 EtOAc:hexanes). ${ }^{1} \mathrm{H}$ NMR (500 $\left.\mathrm{MHz}, \mathrm{CDCl}_{3}\right): \delta 8.15(\mathrm{~d}, J=10 \mathrm{~Hz}, 2 \mathrm{H}), 7.92(\mathrm{~d}, J=5 \mathrm{~Hz}, 2 \mathrm{H}), 7.68(\mathrm{t}, J=15 \mathrm{~Hz}, 1 \mathrm{H}), 7.46-$ $7.53(\mathrm{~m}, 4 \mathrm{H}), 5.57(\mathrm{~s}, 2 \mathrm{H}), 1.36(\mathrm{~s}, 9 \mathrm{H}),{ }^{13} \mathrm{C} \mathrm{NMR}\left(100 \mathrm{MHz}, \mathrm{CDCl}_{3}\right): \delta$ 191.8, 166.2, 157.9, 133.4, 131.9, 130.1, 129.6, 128.5, 127.9, 125.9, 66.5, 35.4, 31.2; HRMS (EI) m/z: $[\mathrm{M}+\mathrm{Na}]^{+}$ Calcd for $\mathrm{C}_{19} \mathrm{H}_{20} \mathrm{O}_{3} \mathrm{Na} 319.1305$; found 319.1306.

2-(4-Fluorophenyl)-2-oxoethyl benzoate $(16)^{6}$<smiles>O=C(COC(=O)c1ccccc1)c1ccc(F)cc1</smiles>

White solid, yield $77 \mathrm{mg}(60 \%), \mathrm{R}_{f} 0.48$ (1:9 EtOAc:hexanes). ${ }^{1} \mathrm{H}$ NMR (500 $\mathrm{MHz}, \mathrm{CDCl}_{3}$ ): $\delta 8.13(\mathrm{~d}, J=10 \mathrm{~Hz}, 2 \mathrm{H}), 7.93-8.01(\mathrm{~m}, 2 \mathrm{H}), 7.59(\mathrm{t}, J=10 \mathrm{~Hz}, 1 \mathrm{H}), 7.45-7.48(\mathrm{~m}, 2 \mathrm{H}), 7.15-$ $7.18(\mathrm{~m}, 2 \mathrm{H}), 5.53(\mathrm{~s}, 2 \mathrm{H})$.

2-(4-Bromophenyl)-2-oxoethyl benzoate (17)<smiles>O=C(COC(=O)c1ccccc1)c1ccc(Br)cc1</smiles> 
White solid, yield $93 \mathrm{mg}$ (58\%), $\mathrm{R}_{f} 0.51$ (1:9 EtOAc:hexanes). ${ }^{1} \mathrm{H}$ NMR (500 MHz, $\mathrm{CDCl}_{3}$ ): $\delta 8.13(\mathrm{~d}, J=10 \mathrm{~Hz}, 2 \mathrm{H}), 7.83(\mathrm{~d}, J=5 \mathrm{~Hz}, 2 \mathrm{H}), 7.65(\mathrm{~d}, J=10 \mathrm{~Hz}, 2 \mathrm{H}), 7.62-7.59(\mathrm{~m}, 1 \mathrm{H})$, 7.49-7.46 (m, 2H), $5.52(\mathrm{~s}, 2 \mathrm{H})$.

\section{2-(3-Bromo-4-fluorophenyl)-2-oxoethyl benzoate (18)}<smiles>O=C(COC(=O)c1ccccc1)c1ccc(F)c(Br)c1</smiles>

Off white solid, yield $92 \mathrm{mg}(55 \%), \mathrm{mp}=93-95{ }^{\circ} \mathrm{C}, \mathrm{R}_{f} 0.42$ (1:9 EtOAc:hexanes). ${ }^{1} \mathrm{H}$ NMR $\left(400 \mathrm{MHz}, \mathrm{CDCl}_{3}\right): \delta 8.21(\mathrm{dd}, J=8,4 \mathrm{~Hz}, 1 \mathrm{H}), 8.12-8.14(\mathrm{~m}, 2 \mathrm{H}), 7.93(\mathrm{~m}, 1 \mathrm{H}), 7.58(\mathrm{t}, J=$ $12 \mathrm{~Hz}, 1 \mathrm{H}), 7.46-7.50(\mathrm{~m}, 2 \mathrm{H}), 7.22-7.27(\mathrm{~m}, 1 \mathrm{H}), 5.51(\mathrm{~s}, 2 \mathrm{H}) ;{ }^{13} \mathrm{C} \mathrm{NMR}\left(100 \mathrm{MHz}, \mathrm{CDCl}_{3}\right)$ : $\delta 189.9,166.0,162.5(\mathrm{~d}, J=250 \mathrm{~Hz}), 134.0,133.6,131.9,130.1,129.2 .129 .1,128.6,117.18$ (d, $J=23 \mathrm{~Hz}), 110.34(\mathrm{~d}, J=22 \mathrm{~Hz}), 66.0$. HRMS (EI) $\mathrm{m} / \mathrm{z}:[\mathrm{M}+\mathrm{Na}]^{+}$Calcd for $\mathrm{C}_{15} \mathrm{H}_{10} \mathrm{O}_{3} \mathrm{BrFNa} 358.9690$; found 358.9690.

\section{2-(4-Nitrophenyl)-2-oxoethyl benzoate (19) ${ }^{6}$}<smiles>O=C(COC(=O)c1ccccc1)c1ccc([N+](=O)[O-])cc1</smiles>

Yellow solid, yield $86 \mathrm{mg}(60 \%), \mathrm{R}_{f} 0.40$ (1:9 EtOAc:hexanes). ${ }^{1} \mathrm{H}$ NMR (500 MHz, $\left.\mathrm{CDCl}_{3}\right)$ : $\delta 8.34(\mathrm{~d}, J=10 \mathrm{~Hz}, 2 \mathrm{H}), 8.20(\mathrm{~d}, J=10 \mathrm{~Hz}, 2 \mathrm{H}), 7.66(\mathrm{t}, J=10 \mathrm{~Hz}, 1 \mathrm{H}), 7.67(\mathrm{~d}, J=10 \mathrm{~Hz}$, 2H), $7.37(\mathrm{~d}, J=15 \mathrm{~Hz}, 2 \mathrm{H}), 5.74(\mathrm{~s}, 2 \mathrm{H})$.

2-Oxo-2-phenylethyl 2-(4-methoxyphenyl)acetate (20)<smiles>COc1ccc(CC(=O)OCC(=O)c2ccccc2)cc1</smiles> 
White solid, yield $100 \mathrm{mg}$ (70\%), $\mathrm{R}_{f} 0.48$ (1:9 EtOAc:hexanes). ${ }^{1} \mathrm{H}$ NMR (400 MHz, $\mathrm{CDCl}_{3}$ ): $\delta 7.88(\mathrm{dd}, J=8,4 \mathrm{~Hz}, 2 \mathrm{H}), 7.58(\mathrm{t}, J=8 \mathrm{~Hz}, 1 \mathrm{H}), 7.44-7.47(\mathrm{~m}, 2 \mathrm{H}), 7.26(\mathrm{~d}, J=8 \mathrm{~Hz}, 2 \mathrm{H})$, $6.87(\mathrm{~d}, J=8 \mathrm{~Hz}, 2 \mathrm{H}), 5.33(\mathrm{~s}, 2 \mathrm{H}), 3.78(\mathrm{~s}, 3 \mathrm{H}), 3.75(\mathrm{~s}, 2 \mathrm{H})$.

\section{1-Benzyl 4-(2-oxo-2-phenylethyl) piperidine-1,4-dicarboxylate (21)}<smiles>O=C(COC(=O)C1CCCN(C(=O)OCc2ccccc2)C1)c1ccccc1</smiles>

White solid, yield $128 \mathrm{mg}(67 \%), \mathrm{mp}=85-87{ }^{\circ} \mathrm{C}, \mathrm{R}_{f} 0.41$ (1:9 EtOAc:hexanes). ${ }^{1} \mathrm{H}$ NMR (500 $\left.\mathrm{MHz}, \mathrm{CDCl}_{3}\right): \delta 7.89(\mathrm{~d}, J=5 \mathrm{~Hz}, 2 \mathrm{H}), 7.60(\mathrm{t}, J=10 \mathrm{~Hz}, 1 \mathrm{H}), 7.47-7.50(\mathrm{~m}, 2 \mathrm{H}), 7.30-7.38$ (m, 5H), 5.26-5.38 (m, 2H), 5.12-5.17 (m, 2H), $4.27(\mathrm{~s}, 1 \mathrm{H}), 4.01(\mathrm{~d}, J=15 \mathrm{~Hz}, 1 \mathrm{H}), 3.24(\mathrm{~s}$, 1H), 2.93-2.99 (m, 1H), $2.67(\mathrm{~s}, 1 \mathrm{H}), 2.17-2.19(\mathrm{~m}, 1 \mathrm{H}), 1.74-1.82(\mathrm{~m}, 2 \mathrm{H}), 1.54-1.56(\mathrm{~m}, 1 \mathrm{H})$; ${ }^{13} \mathrm{C}$ NMR (100 MHz, $\left.\mathrm{CDCl}_{3}\right): \delta 191.8,172.7,155.3,136.8,134.1,134.0,128.9,128.5,128.1$, 128.0, 127.8, 66.2, 66.0, 45.7, 44.3, 41.1, 27.4, 24.3; HRMS (EI) m/z: [M + Na $]^{+}$Calcd for $\mathrm{C}_{22} \mathrm{H}_{23} \mathrm{O}_{5} \mathrm{NNa}$ 404.1468; found 404.1473.

\section{2-Oxo-2-(p-tolyl)ethyl cyclohex-1-ene-1-carboxylate (22)}<smiles>Cc1ccc(C(=O)COC(=O)C2=CCCCC2)cc1</smiles>

Colorless semi solid, yield $83 \mathrm{mg}$ (64\%), $\mathrm{R}_{f} 0.55$ (1:9 EtOAc:hexanes). ${ }^{1} \mathrm{H}$ NMR (500 MHz, $\left.\mathrm{CDCl}_{3}\right): \delta 7.83(\mathrm{~d}, J=8 \mathrm{~Hz}, 2 \mathrm{H}), 7.28(\mathrm{~d}, J=8 \mathrm{~Hz}, 2 \mathrm{H}), 7.15-7.16(\mathrm{~m}, 1 \mathrm{H}), 5.37(\mathrm{~s}, 2 \mathrm{H}), 2.42$ (s, 3H), 2.37-2.39 (m, 2H), $2.28(\mathrm{dd}, J=10,5 \mathrm{~Hz}, 2 \mathrm{H}), 1.56-1.70(\mathrm{~m}, 4 \mathrm{H}) ;{ }^{13} \mathrm{C}$ NMR (100 $\left.\mathrm{MHz}, \mathrm{CDCl}_{3}\right): \delta 192.3,167.0,144.8,141.3,132.2,129.6,128.0,126.7,65.9,26.0,24.2,22.1$, 21.9, 21.5; HRMS (EI) m/z: [M + Na] ${ }^{+}$Calcd for $\mathrm{C}_{16} \mathrm{H}_{8} \mathrm{O}_{3} \mathrm{Na} 281.1148$; found 281.1151. 
2-Oxo-2-phenylethyl 2-nitrobenzoate (23) ${ }^{6}$<smiles>O=C(COC(=O)c1ccccc1[N+](=O)[O-])c1ccccc1</smiles>

Yellow solid, yield $93 \mathrm{mg}$ (65\%), $\mathrm{R}_{f} 0.41$ (1:9 EtOAc:hexanes). ${ }^{1} \mathrm{H}$ NMR (400 MHz, $\mathrm{CDCl}_{3}$ ): $\delta$ 7.93-7.97 (m, 4H), 7.71-7.73 (m, 3H), 7.48-7.53 (m, 2H), $5.59(\mathrm{~s}, 2 \mathrm{H})$.

\section{1-Phenyl-2-(p-tolylamino)ethan-1-one (24) ${ }^{9}$}<smiles>Cc1ccc(NCC(=O)c2ccccc2)cc1</smiles>

Yellow liquid, yield $62 \mathrm{mg}(55 \%), \mathrm{R}_{f} 0.42$ (1:9 EtOAc:hexanes). ${ }^{1} \mathrm{H}$ NMR (400 MHz, $\mathrm{CDCl}_{3}$ ): $\delta 8.02(\mathrm{~d}, J=8 \mathrm{~Hz}, 2 \mathrm{H}), 7.61(\mathrm{t}, J=8 \mathrm{~Hz}, 1 \mathrm{H}), 7.53-7.50(\mathrm{~m}, 2 \mathrm{H}), 7.04(\mathrm{~d}, J=8 \mathrm{~Hz}, 2 \mathrm{H}), 6.65$ $(\mathrm{d}, J=8 \mathrm{~Hz}, 2 \mathrm{H}), 4.61(\mathrm{~s}, 2 \mathrm{H}), 2.26(\mathrm{~s}, 3 \mathrm{H})$.

\section{2-Morpholino-1-phenylpropan-1-one (25) ${ }^{9}$}<smiles>CC(C(=O)c1ccccc1)N1CCOCC1</smiles>

Yellow liquid, yield $86 \mathrm{mg}$ (78\%), $\mathrm{R}_{f} 0.22$ (1:4 EtOAc:hexanes). ${ }^{1} \mathrm{H}$ NMR (500 MHz, $\left.\mathrm{CDCl}_{3}\right)$ : $\delta 8.08(\mathrm{~d}, J=10 \mathrm{~Hz}, 2 \mathrm{H}), 7.55(\mathrm{~d}, J=10 \mathrm{~Hz}, 1 \mathrm{H}), 7.47-7.44(\mathrm{~m}, 2 \mathrm{H}), 4.09-4.05(\mathrm{~m}, 1 \mathrm{H}), 3.70-$ $3.67(\mathrm{~m}, 4 \mathrm{H}), 2.63-2.56(\mathrm{~m}, 4 \mathrm{H}), 1.29(\mathrm{~d}, J=5 \mathrm{~Hz}, 3 \mathrm{H})$.

\section{2-Azido-1-(p-tolyl)ethan-1-ol (26)}<smiles>Cc1ccc(C(O)CN)cc1</smiles> 
Colourless liquid, yield $69 \mathrm{mg}$ (78\%), $\mathrm{R}_{\mathrm{f}} 0.41$ (1:9 EtOAc:hexanes). ${ }^{1} \mathrm{H}$ NMR (500 MHz, $\left.\mathrm{CDCl}_{3}\right): \delta 7.27(\mathrm{~d}, J=5 \mathrm{~Hz}, 2 \mathrm{H}), 7.20(\mathrm{~d}, J=5 \mathrm{~Hz}, 2 \mathrm{H}), 4.85(\mathrm{t}, J=10 \mathrm{~Hz}, 1 \mathrm{H}), 3.45(\mathrm{~d}, J=$ $15 \mathrm{~Hz}, 2 \mathrm{H}), 2.40(\mathrm{br}, 1 \mathrm{H}), 2.36(\mathrm{~s}, 3 \mathrm{H})$.

\section{2-Azido-1-(4-fluorophenyl)ethan-1-ol (27) ${ }^{7}$}<smiles>NCC(O)c1ccc(F)cc1</smiles>

Yellow liquid, yield $55 \mathrm{mg}(61 \%), \mathrm{R}_{\mathrm{f}} 0.40$ (1:9 EtOAc:hexanes). ${ }^{1} \mathrm{H}$ NMR (500 MHz, $\left.\mathrm{CDCl}_{3}\right)$ : $\delta 7.52(\mathrm{~d}, J=10 \mathrm{~Hz}, 2 \mathrm{H}), 7.26(\mathrm{~d}, J=5 \mathrm{~Hz}, 2 \mathrm{H}), 4.84(\mathrm{t}, J=10 \mathrm{~Hz}, 1 \mathrm{H}), 3.45(\mathrm{~d}, J=5 \mathrm{~Hz}$, 2H), 2.41 (br, 1H).

2-Azido-1-(4-chlorophenyl)propan-1-ol (28)<smiles>[C-]C(N)C(O)c1ccc(Cl)cc1</smiles>

Obtained as mixture of cis and trans isomers. Colourless liquid, yield $61 \mathrm{mg}(58 \%), \mathrm{R}_{f} 0.41$ (1:9 EtOAc:hexanes). ${ }^{1} \mathrm{H}$ NMR (500 MHz, $\left.\mathrm{CDCl}_{3}\right): \delta 7.35$ (d, $\left.J=10 \mathrm{~Hz}, 2 \mathrm{H}\right), 7.29(\mathrm{~d}, J=10$ $\mathrm{Hz}, 2 \mathrm{H}), 4.43(\mathrm{~d}, J=5 \mathrm{~Hz}, 1 \mathrm{H}), 3.62-3.73(\mathrm{~m}, 1 \mathrm{H}), 2.48(\mathrm{br}, 1 \mathrm{H}), 1.11(\mathrm{~d}, J=5 \mathrm{~Hz}, 3 \mathrm{H}) . ;{ }^{13} \mathrm{C}$ $\operatorname{NMR}\left(100 \mathrm{MHz}, \mathrm{CDCl}_{3}\right): \delta 138.8,134.3,128.8,128.7,128.2,127.9,63.4,62.3,15.9,13.5$. HRMS (EI) m/z: [M - $\left.\mathrm{N}_{2}+\mathrm{H}\right]^{+}$Calcd for $\mathrm{C}_{9} \mathrm{H}_{11} \mathrm{ONCl} 184.0524$; found 184.0525. 


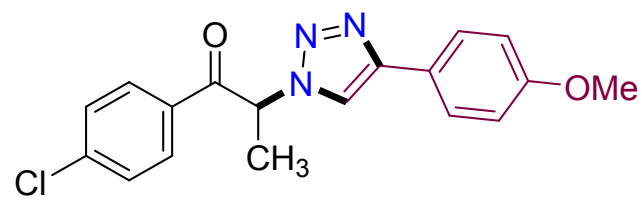

White solid, yield $109 \mathrm{mg}(64 \%), \mathrm{mp}=157-159{ }^{\circ} \mathrm{C}, \mathrm{R}_{f} 0.12$ (1:1 EtOAc:hexanes). ${ }^{1} \mathrm{H}$ NMR $\left(400 \mathrm{MHz}, \mathrm{CDCl}_{3}\right): \delta 7.99(\mathrm{~d}, J=8 \mathrm{~Hz}, 2 \mathrm{H}), 7.87(\mathrm{~s}, 1 \mathrm{H}), 7.76(\mathrm{~d}, J=8 \mathrm{~Hz}, 2 \mathrm{H}), 7.47$ (d, $J=$ $16 \mathrm{~Hz}, 2 \mathrm{H}), 6.95(\mathrm{~d}, J=8 \mathrm{~Hz}, 2 \mathrm{H}), 6.45$ (q, $J=8 \mathrm{~Hz}, 1 \mathrm{H}), 3.84(\mathrm{~s}, 3 \mathrm{H}), 1.85(\mathrm{~d}, J=4 \mathrm{~Hz}, 3 \mathrm{H})$; ${ }^{13} \mathrm{C}$ NMR $\left(100 \mathrm{MHz}, \mathrm{CDCl}_{3}\right): \delta 192.1,158.8,140.2,131.3,129.3,128.6,126.1122 .2,113.3$, 58.4, 54.4, 17.7. HRMS (EI) $\mathrm{m} / \mathrm{z}$ : $[\mathrm{M}+\mathrm{Na}]^{+}$Calcd for $\mathrm{C}_{18} \mathrm{H}_{16} \mathrm{O}_{2} \mathrm{~N}_{3} \mathrm{ClNa}$ 364.0823; found 364.0825 .

2-(4-((5-Bromo-1H-indol-1-yl)methyl)-1H-1,2,3-triazol-1-yl)-1-(p-tolyl)ethan-1-one (30)

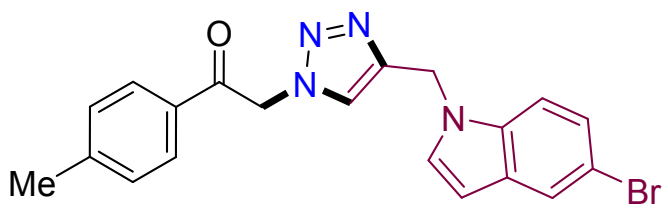

Brown solid, yield $135 \mathrm{mg}(66 \%), \mathrm{mp}=70-72{ }^{\circ} \mathrm{C}, \mathrm{R}_{\mathrm{f}} 0.15$ (7:3 EtOAc: Hexane). ${ }^{1} \mathrm{H}$ NMR (400 $\left.\mathrm{MHz}, \mathrm{CDCl}_{3}\right): \delta 7.60(\mathrm{~d}, J=8 \mathrm{~Hz}, 2 \mathrm{H}), 7.51(\mathrm{~s}, 1 \mathrm{H}), 7.23(\mathrm{br}, 1 \mathrm{H}), 7.04-7.10(\mathrm{~m}, 4 \mathrm{H}), 6.98$ $(\mathrm{s}, 1 \mathrm{H}), 6.22(\mathrm{~s}, 1 \mathrm{H}), 5.49(\mathrm{~s}, 2 \mathrm{H}), 5.23(\mathrm{br}, 2 \mathrm{H}), 2.20(3 \mathrm{H}) ;{ }^{13} \mathrm{C} \mathrm{NMR}\left(100 \mathrm{MHz}, \mathrm{CDCl}_{3}\right): \delta$ 189.6, 146.0, 134.6, 131.3, 130.6, 129.9, 129.2, 128.3, 124.8, 123.6, 113.3, 111.2, 101. 7, 55.5, 42.4, 21.9. HRMS (EI) m/z: $[\mathrm{M}+\mathrm{Na}]^{+}$Calcd for $\mathrm{C}_{20} \mathrm{H}_{17} \mathrm{O}_{2} \mathrm{~N}_{4} \mathrm{BrNa} 431.0478$; found 431.0479 . 


\section{REFERENCES}

(1) Fulmer, G. R.; Miller, A. J. M.; Sherden, N. H.; Gottlieb, H. E.; Nudelman, A.; Stoltz, B. M.; Bercaw, J. E.; Goldberg, K. I. NMR Chemical Shifts of Trace Impurities: Common Laboratory Solvents, Organics, and Gases in Deuterated Solvents Relevant to the Organometallic Chemist. Organometallics 2010, 29, 2176-2179.

(2) Brals, J.; Smith, J. D.; Ibrahim, F.; Gallou, F.; Handa, S. Micelle-Enabled Palladium Catalysis for Convenient $S p^{2}-S p^{3}$ Coupling of Nitroalkanes with Aryl Bromides in Water Under Mild Conditions. ACS Catal. 2017, 7, 7245-7250.

(3) Venkat, R. R.; Kumareswaran, R.; Vankar, Y. D. A one step conversion of olefins into $\alpha-$ Azidoketones using azidotrimethylsilanechromium trioxide reagent system. Tetrahedron Lett. 1995, 36, 6751-6754.

(4) Vita, M. V.; Waser, J. Azidation of I-Keto Esters and Silyl Enol Ethers with a Benziodoxole Reagent. Org. Lett. 2013, 15, 3246-3249.

(5) Hossain, A.; Vidyasagar, A.; Eichinger, C.; Lankes, C.; Phan, J.; Rehbein, J.; Reiser, O. Visible-Light-Accelerated Copper(II)-Catalyzed Regio- and Chemoselective Oxo-Azidation of Vinyl Arenes. Angew. Chem., Int. Ed. 2018, 57, 8288-8292.

(6) Reddi, R. N.; Prasad, P. K.; Sudalai, A. I 2 -Catalyzed Regioselective Oxo- and Hydroxyacyloxylation of Alkenes and Enol Ethers: A Facile Access to $\alpha$-Acyloxyketones, Esters, and Diol Derivatives. Org. Lett. 2014, 16, 5674-5677.

(7) Yang, B.; Lu. Z. Visible-Light-Promoted Metal-Free Aerobic Hydroxyazidation of Alkenes. ACS Catal. 2017, 7, 8362-8365.

(8) Ye, A.-H.; Zhang, Y.; Xie, Y.-Y.; Luo, H.-Y.; Dong, J.-W.; Liu, X.- D.; Song, X.-F.; Ding, T.; Chen, Z.-M. TMSCl-Catalyzed Electrophilic Thiocyano Oxyfunctionalization of Alkenes Using N-Thiocyano-dibenzenesulfonimide .Org. Lett. 2019, 21, 5106-5110.

(9) Shinde, M. H.; Kshirsagar, U. A. N-Bromosuccinimide promoted and base switchable one pot synthesis of $\alpha$-imido and $\alpha$-amino ketones from styrenes. Org. Biomol. Chem. 2016, 14, $858-861$. 
12. ${ }^{1} \mathrm{H} \&{ }^{13} \mathrm{C}$ NMR SPECTRA OF COMPOUNDS 2-30

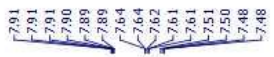
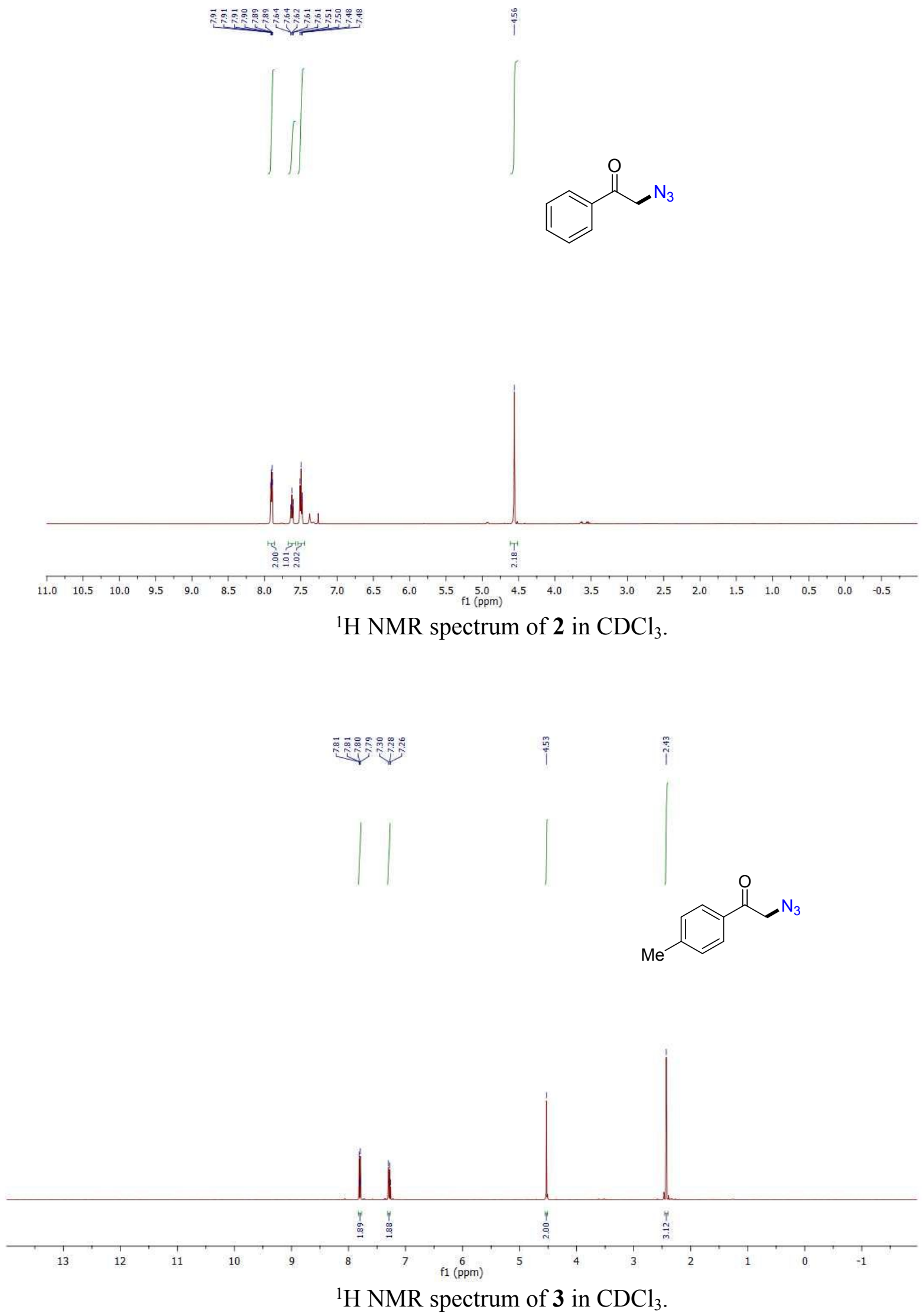

S24 


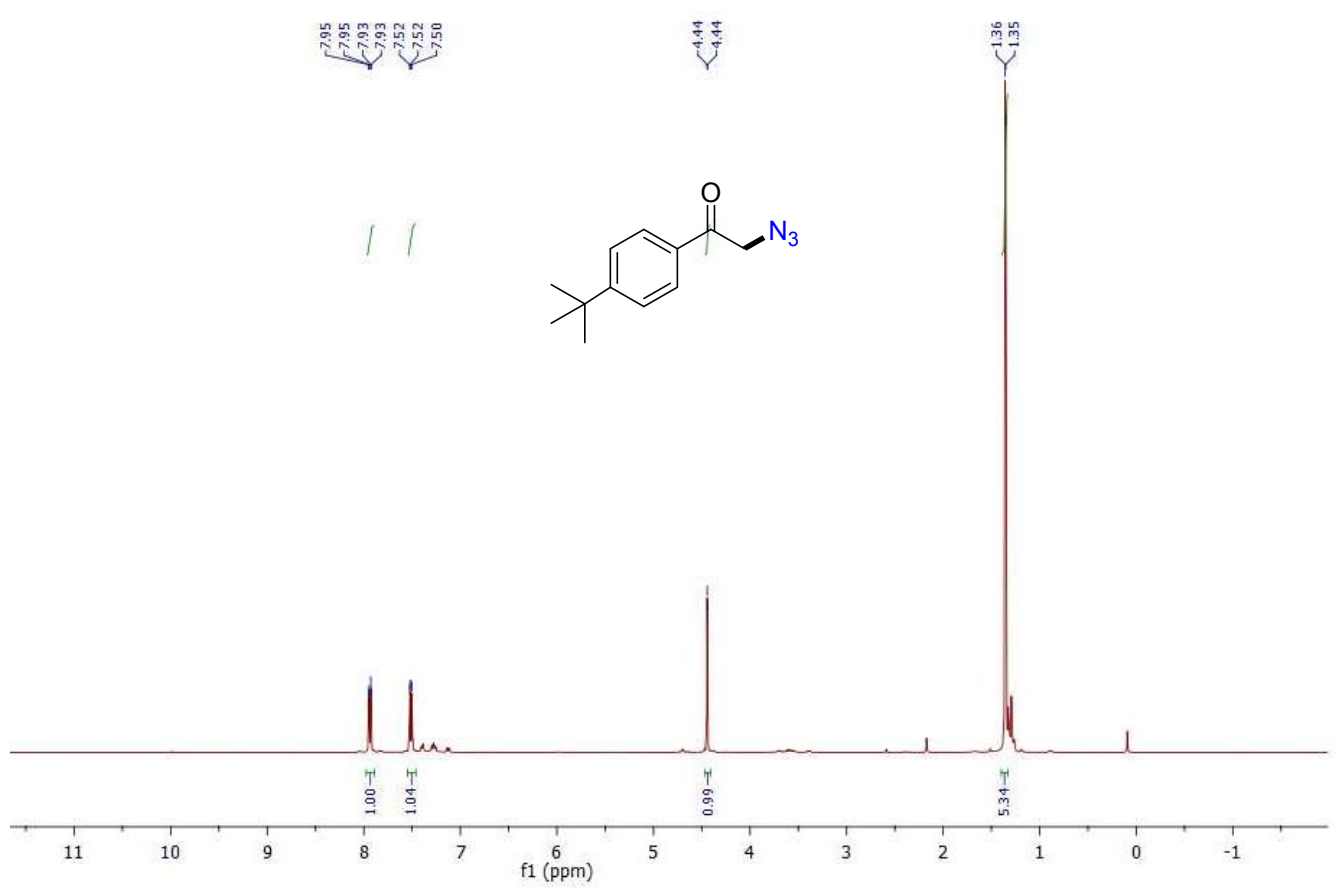

${ }^{1} \mathrm{H}$ NMR spectrum of $\mathbf{4}$ in $\mathrm{CDCl}_{3}$.

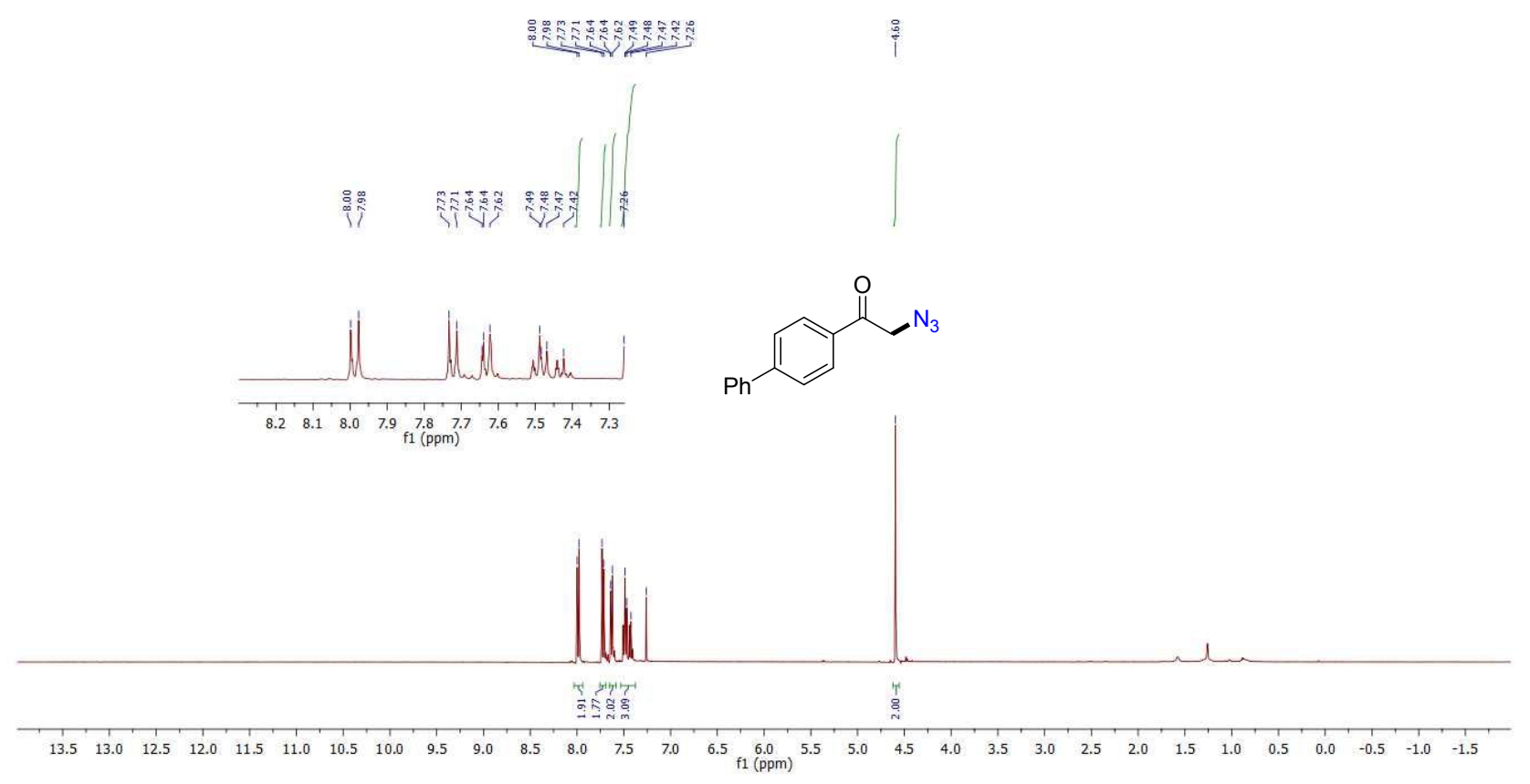

${ }^{1} \mathrm{H} \mathrm{NMR}$ spectrum of 5 in $\mathrm{CDCl}_{3}$. 

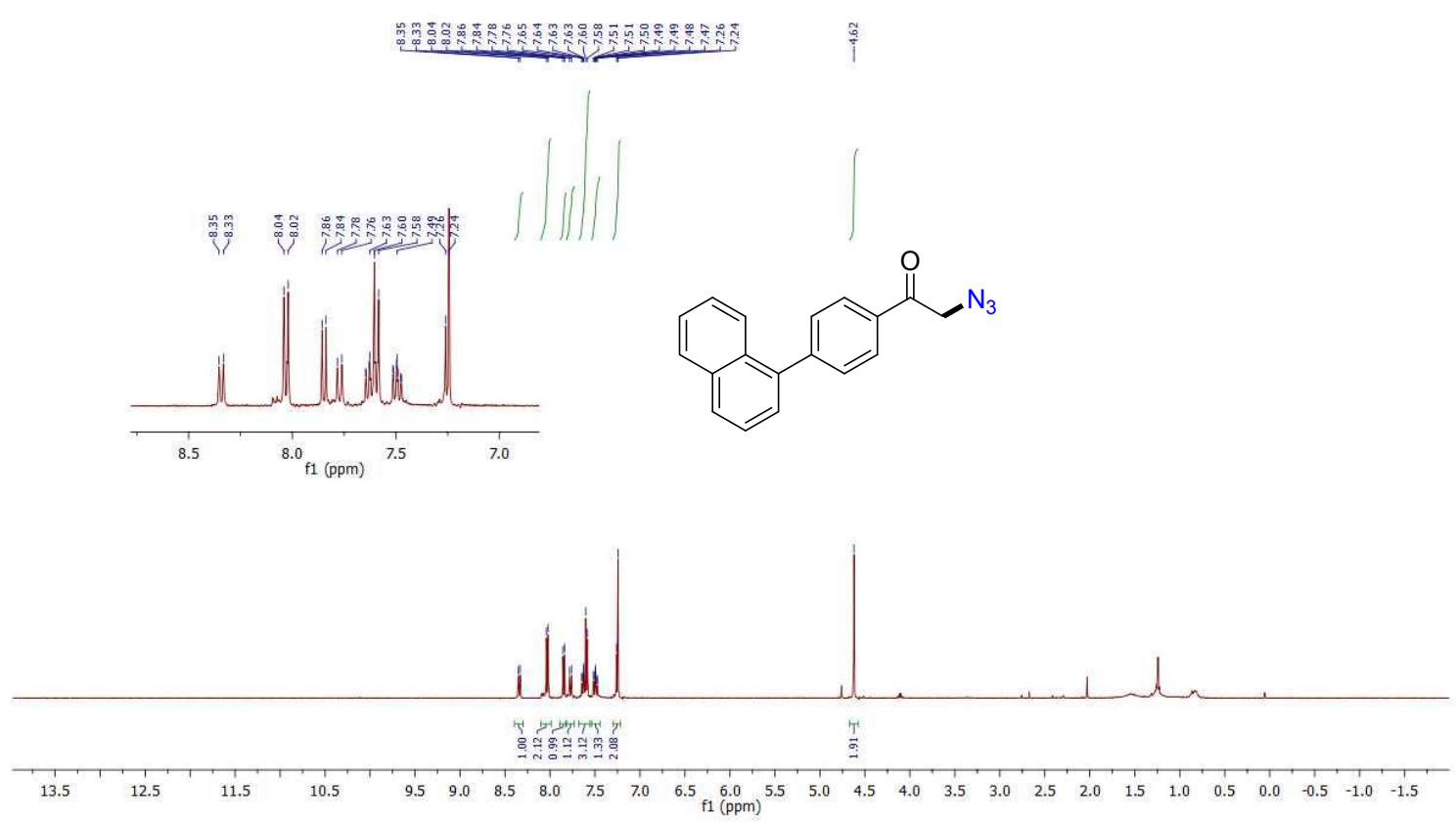

${ }^{1} \mathrm{H}$ NMR spectrum of 6 in $\mathrm{CDCl}_{3}$.

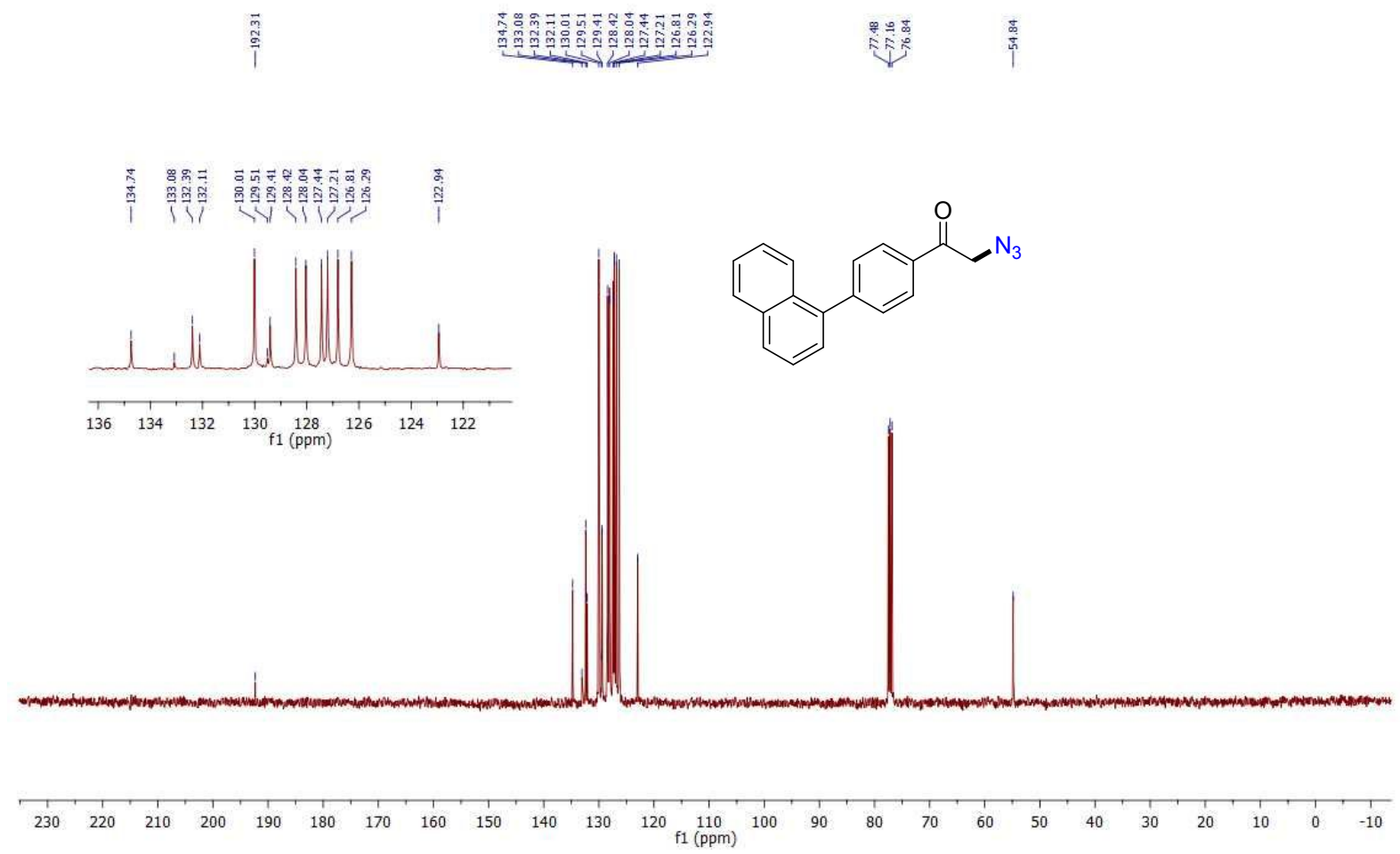

${ }^{13} \mathrm{C}$ NMR spectrum of 6 in $\mathrm{CDCl}_{3}$. 

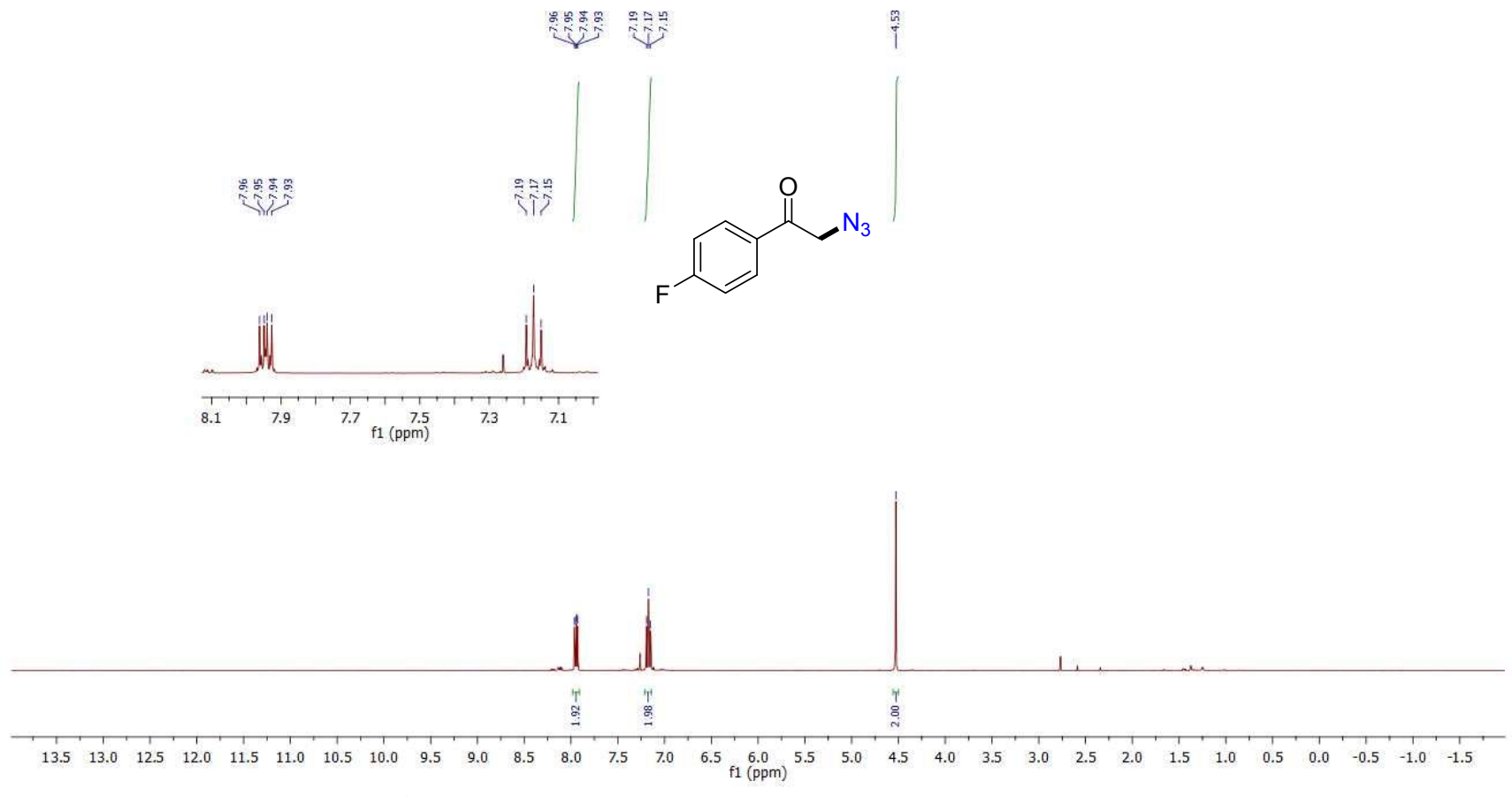

${ }^{1} \mathrm{H}$ NMR spectrum of 7 in $\mathrm{CDCl}_{3}$. 

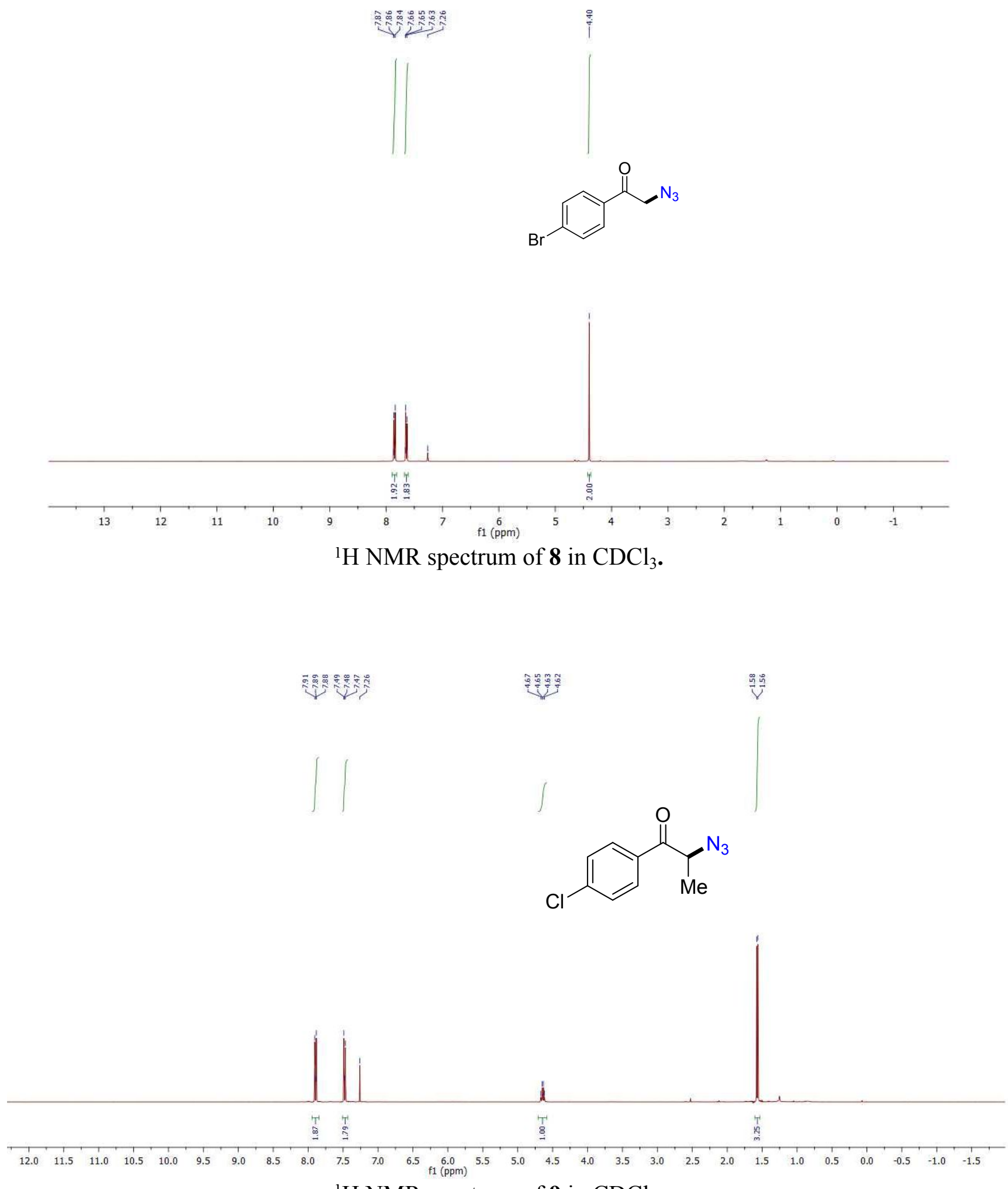

${ }^{1} \mathrm{H}$ NMR spectrum of 9 in $\mathrm{CDCl}_{3}$. 

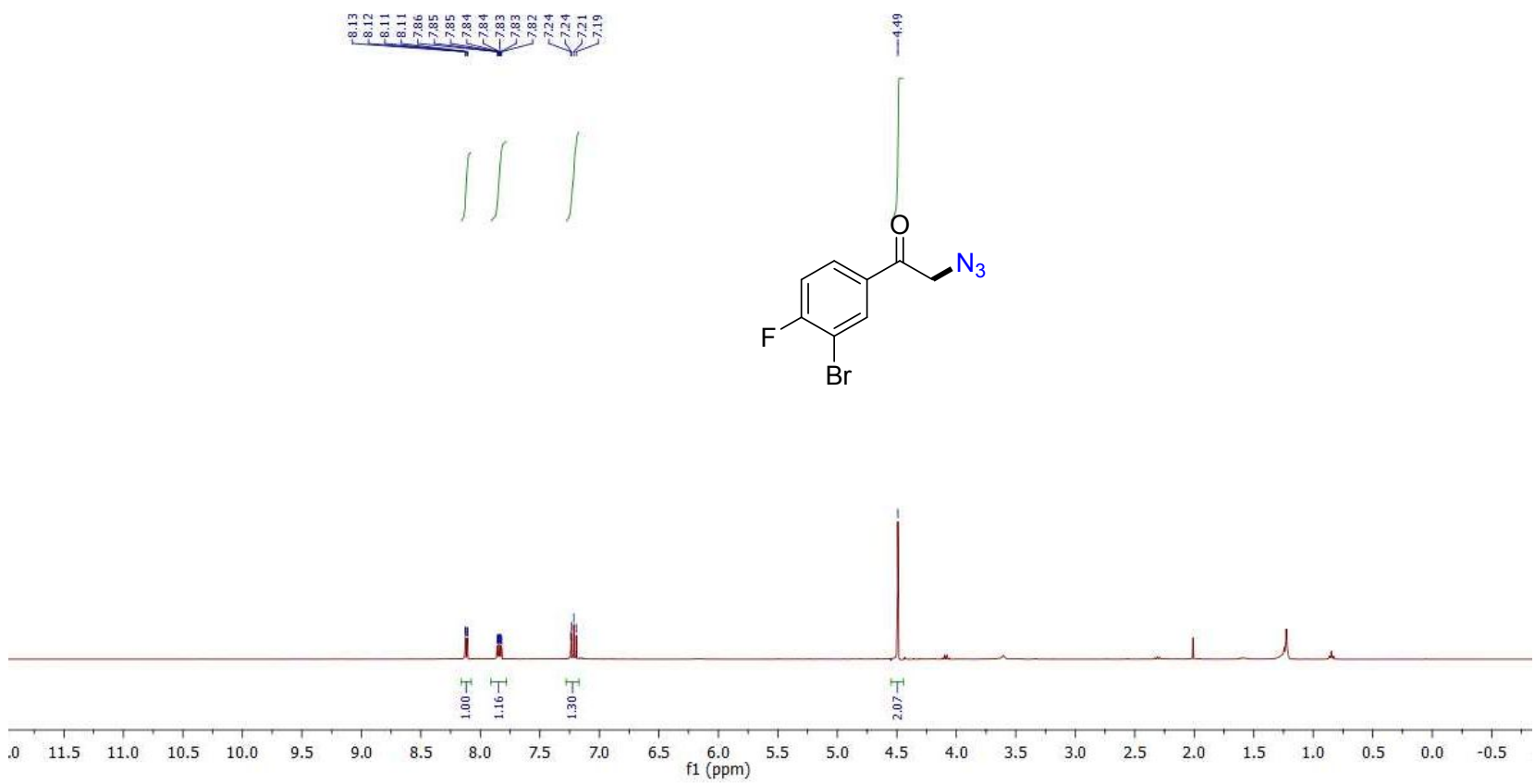

${ }^{1} \mathrm{H} \mathrm{NMR}$ spectrum of $\mathbf{1 0}$ in $\mathrm{CDCl}_{3}$.

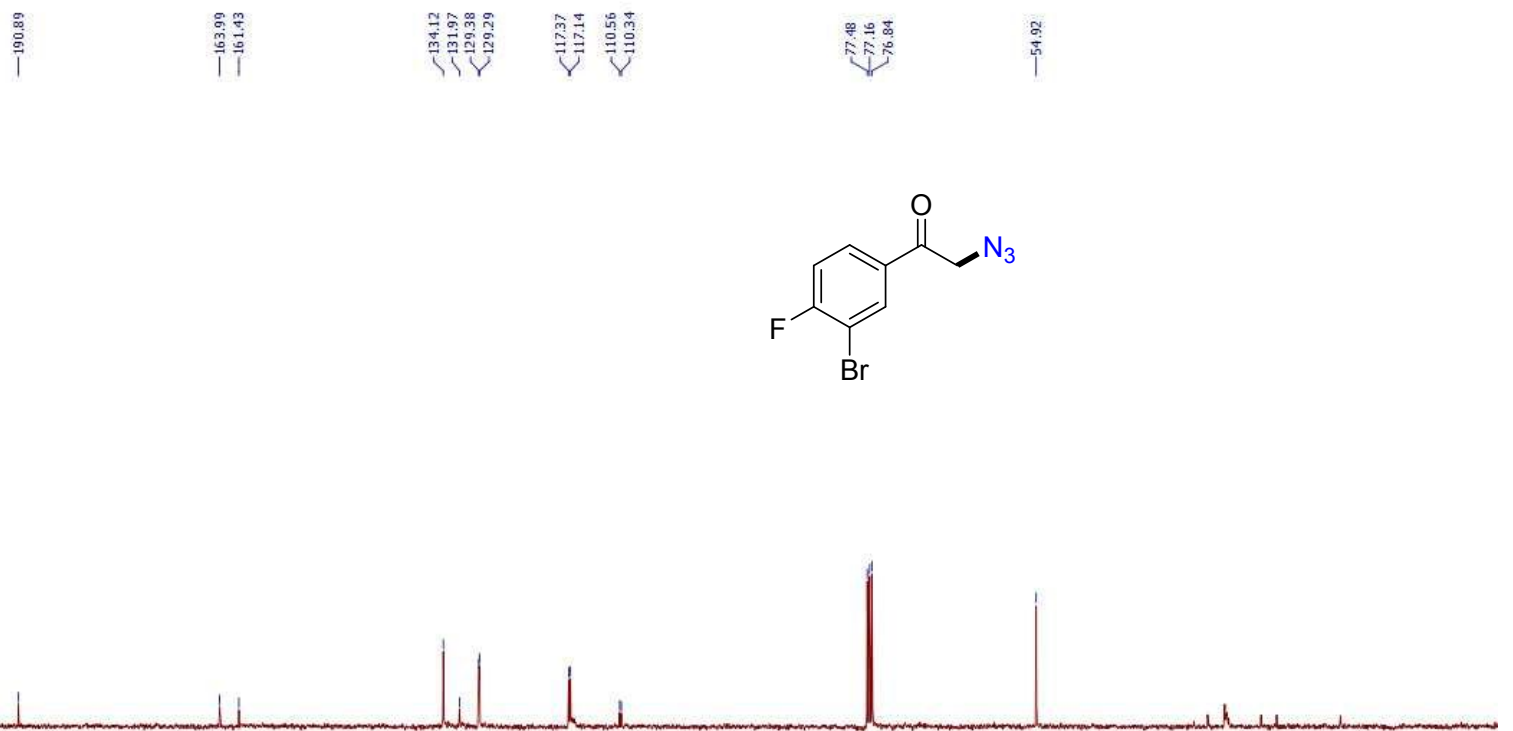

$\begin{array}{llllllllllllllllllllllllllll}230 & 220 & 210 & 200 & 190 & 180 & 170 & 160 & 150 & 140 & 130 & 120 & 110 & 100 & 90 & 80 & 70 & 60 & 50 & 40 & 30 & 20 & 10 & 0\end{array}$

${ }^{13} \mathrm{C}$ NMR spectrum of $\mathbf{1 0}$ in $\mathrm{CDCl}_{3}$. 


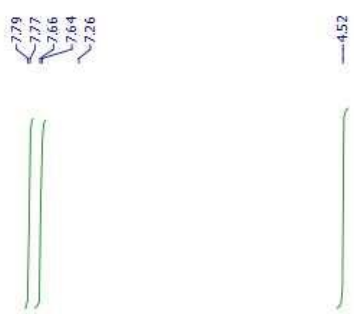<smiles>NCC(=O)c1ccc(Cl)cc1</smiles>

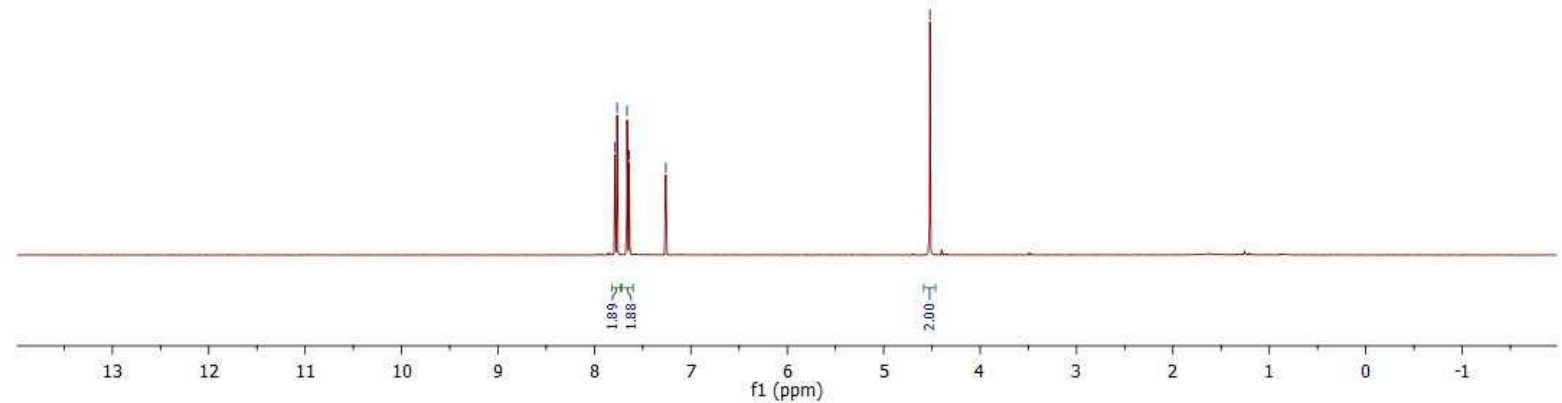

${ }^{1} \mathrm{H}$ NMR spectrum of $\mathbf{1 1}$ in $\mathrm{CDCl}_{3}$.
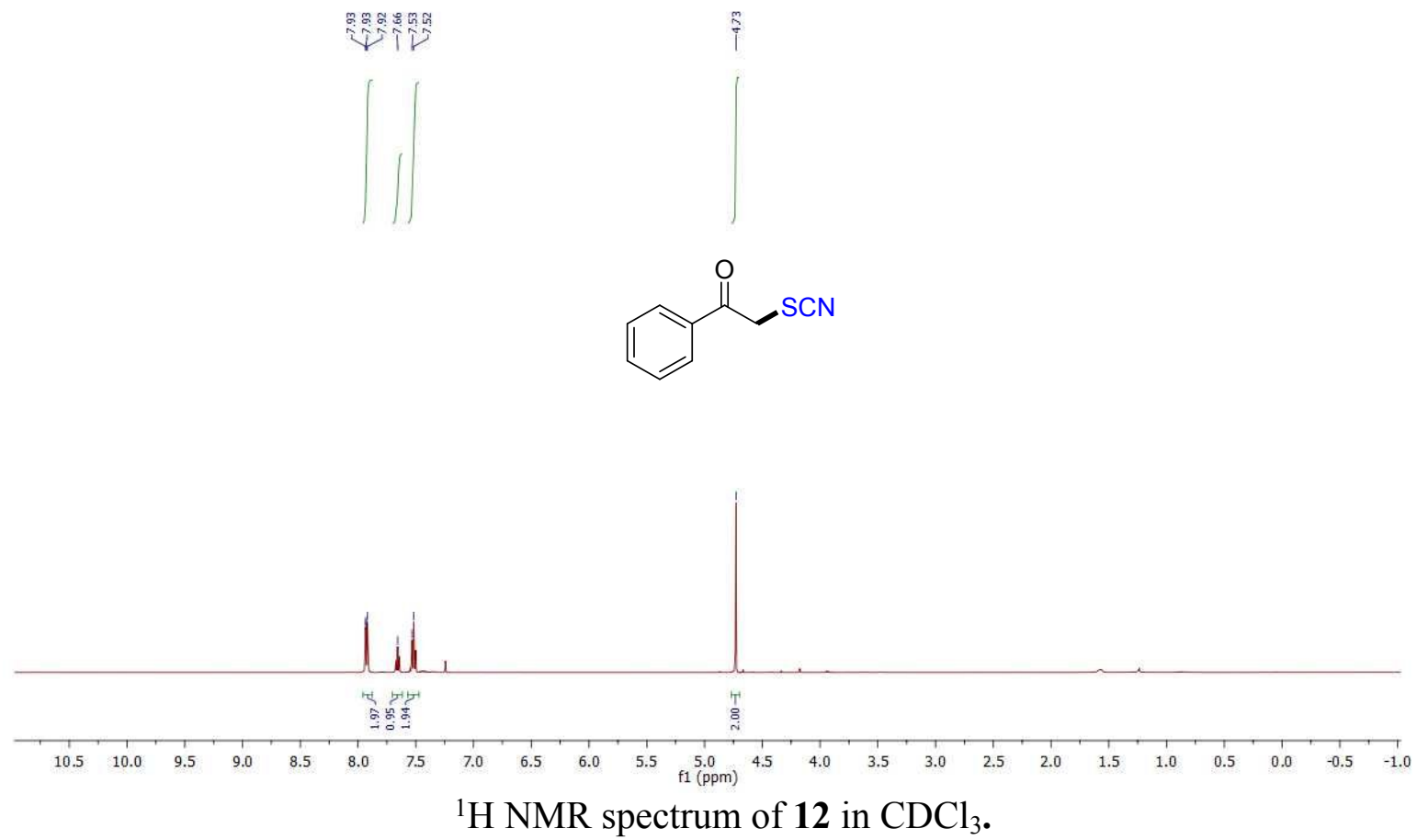


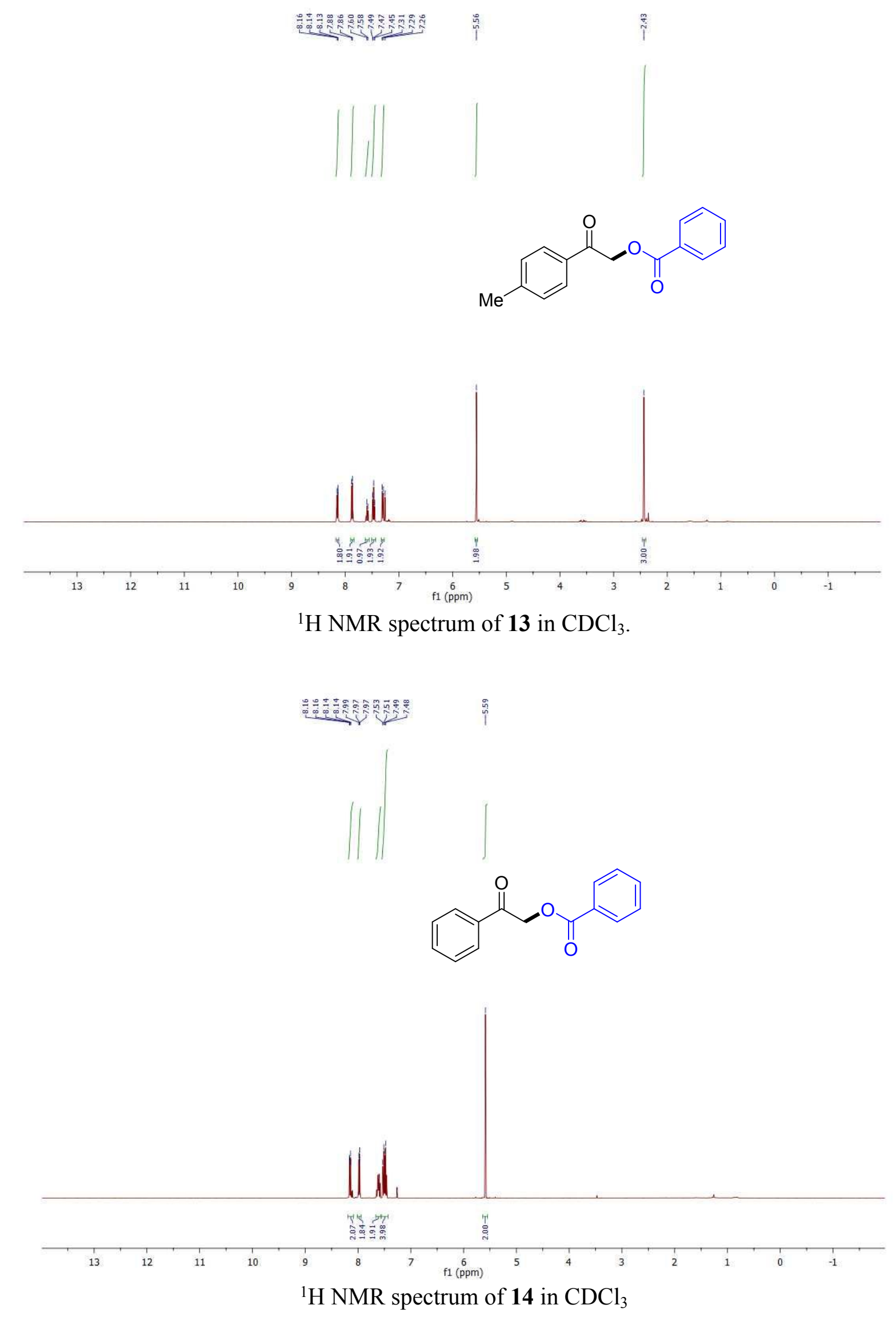




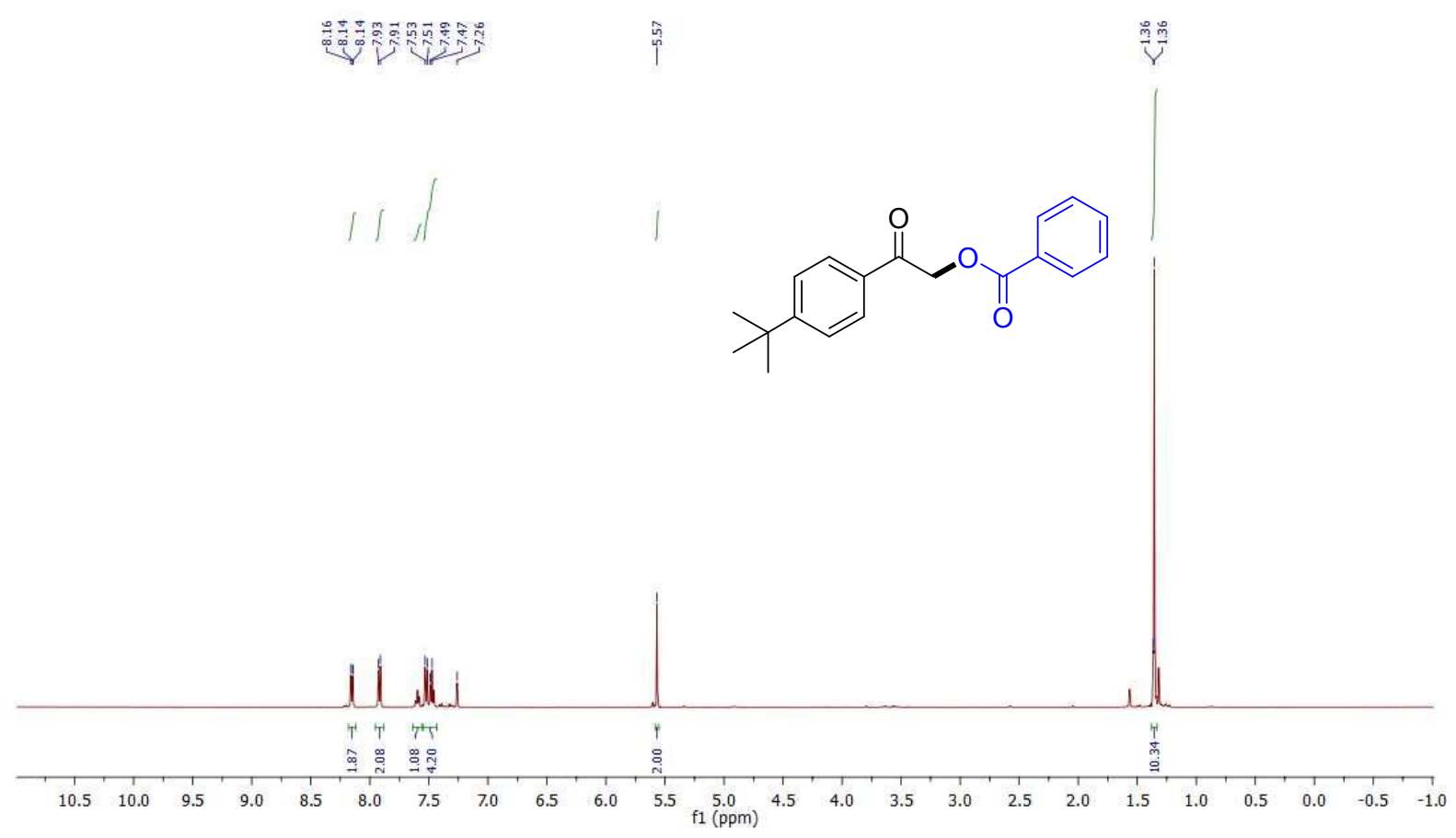

${ }^{1} \mathrm{H}$ NMR spectrum of $\mathbf{1 5}$ in $\mathrm{CDCl}_{3}$.

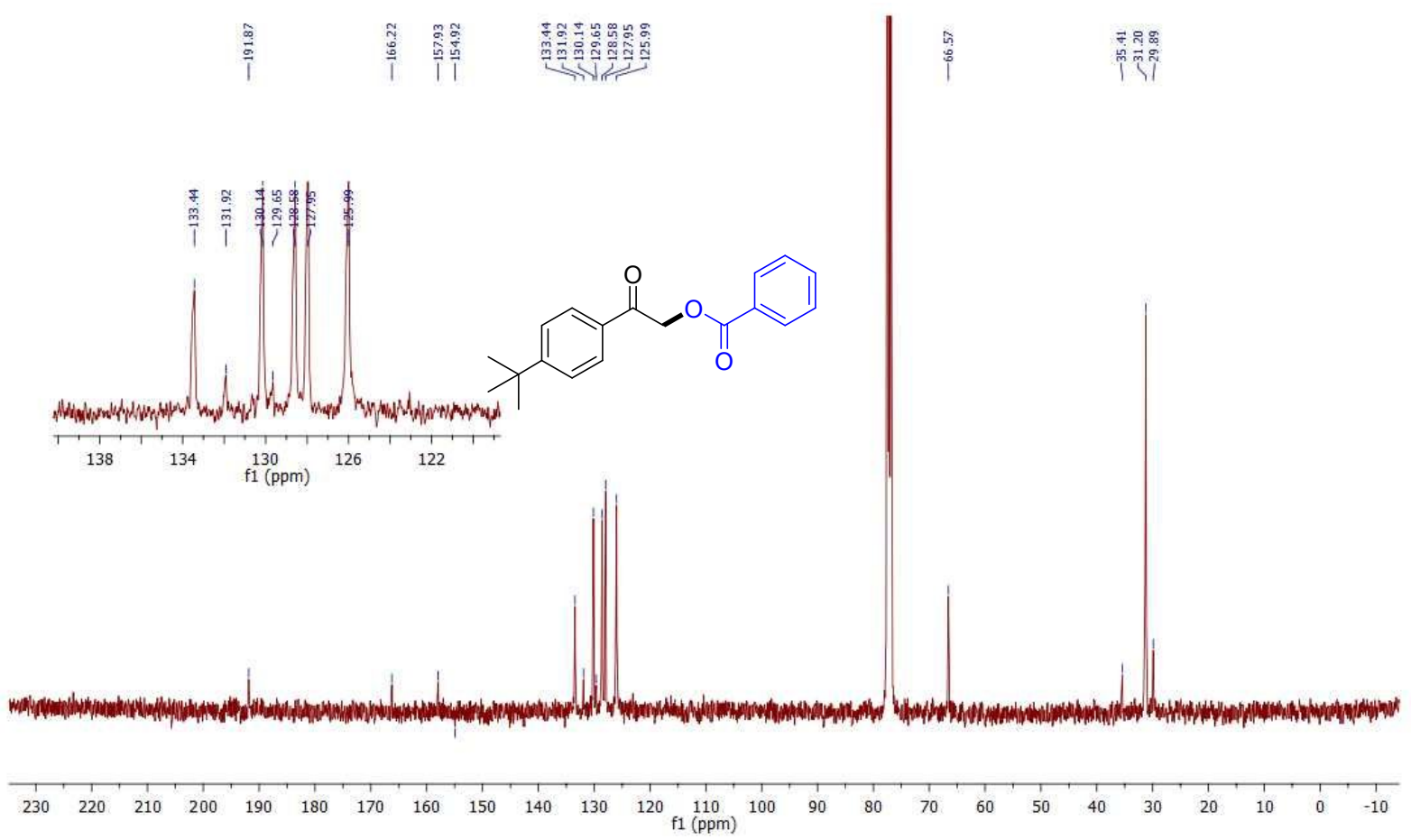

${ }^{13} \mathrm{C}$ NMR spectrum of 15 in $\mathrm{CDCl}_{3}$. 


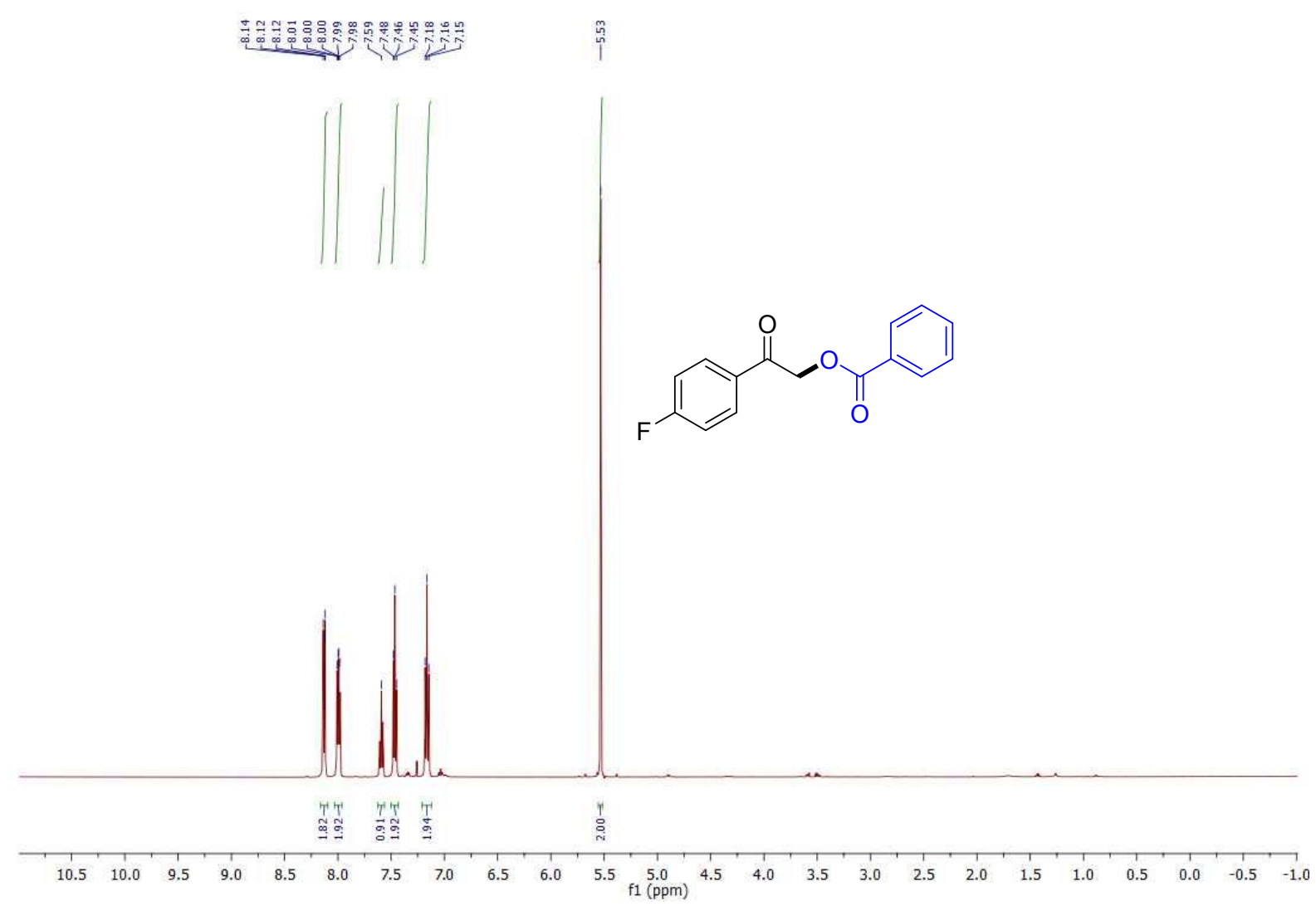

${ }^{1} \mathrm{H}$ NMR spectrum of $\mathbf{1 6}$ in $\mathrm{CDCl}_{3}$.

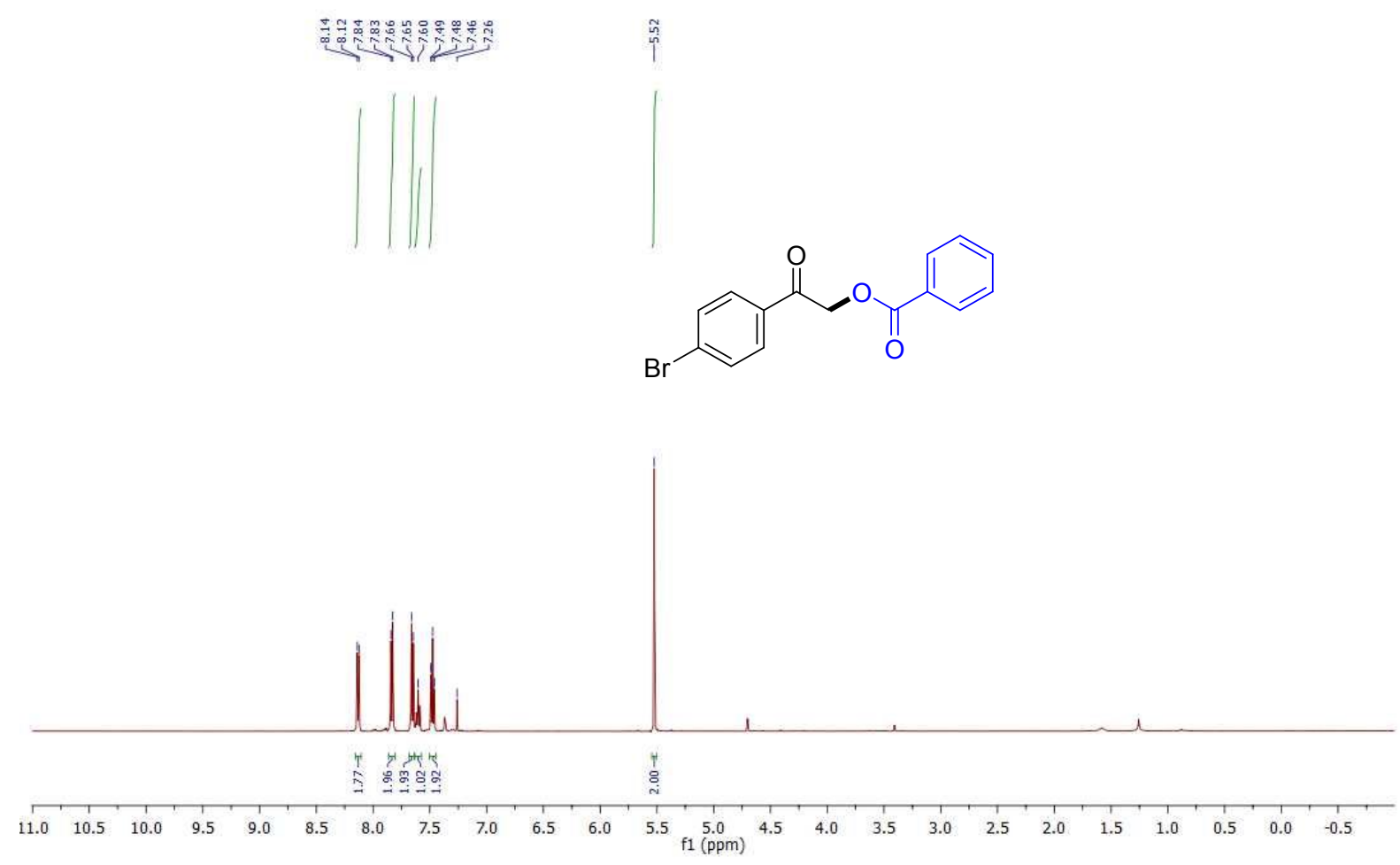

${ }^{1} \mathrm{H}$ NMR spectrum of 17 in $\mathrm{CDCl}_{3}$. 


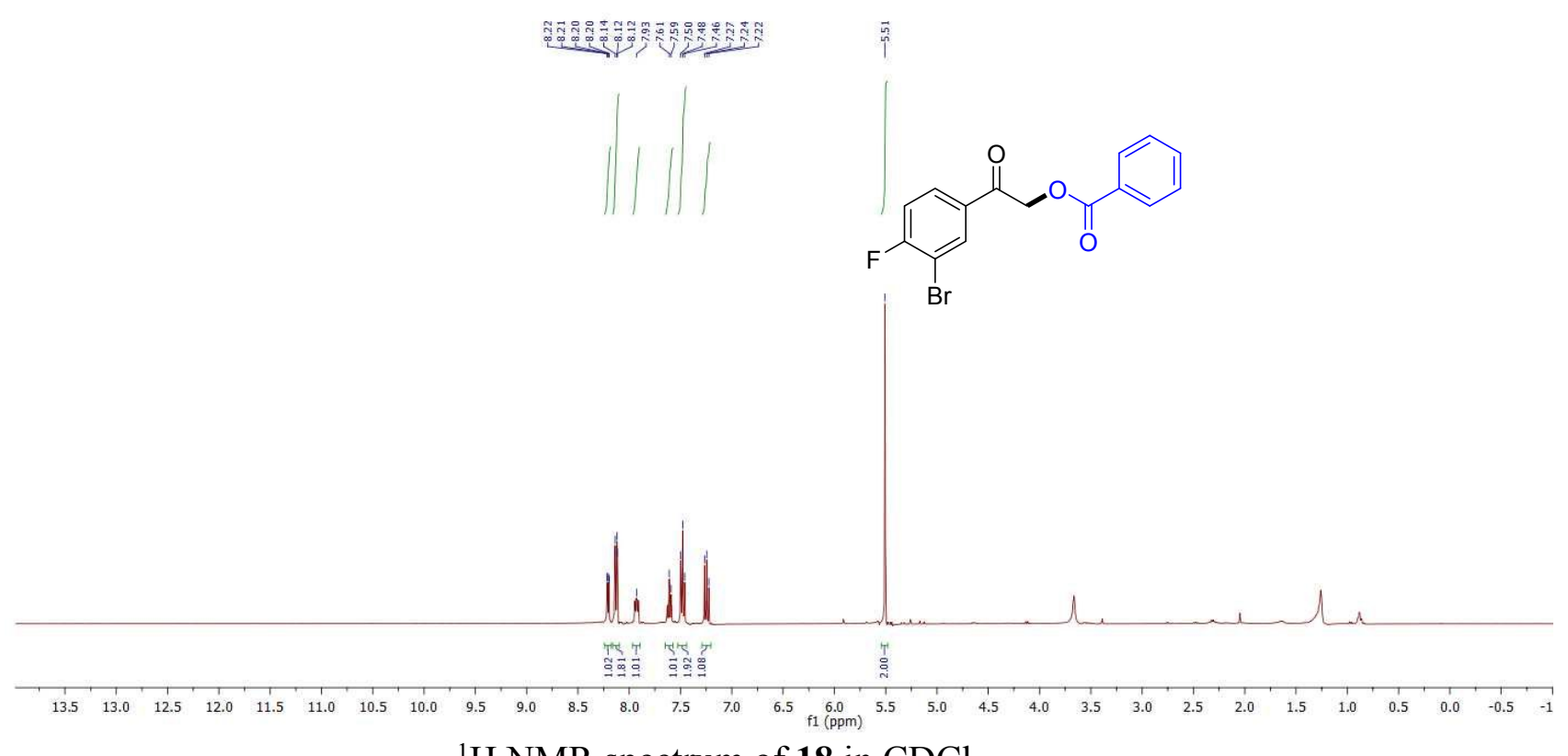

${ }^{1} \mathrm{H}$ NMR spectrum of $\mathbf{1 8}$ in $\mathrm{CDCl}_{3}$.
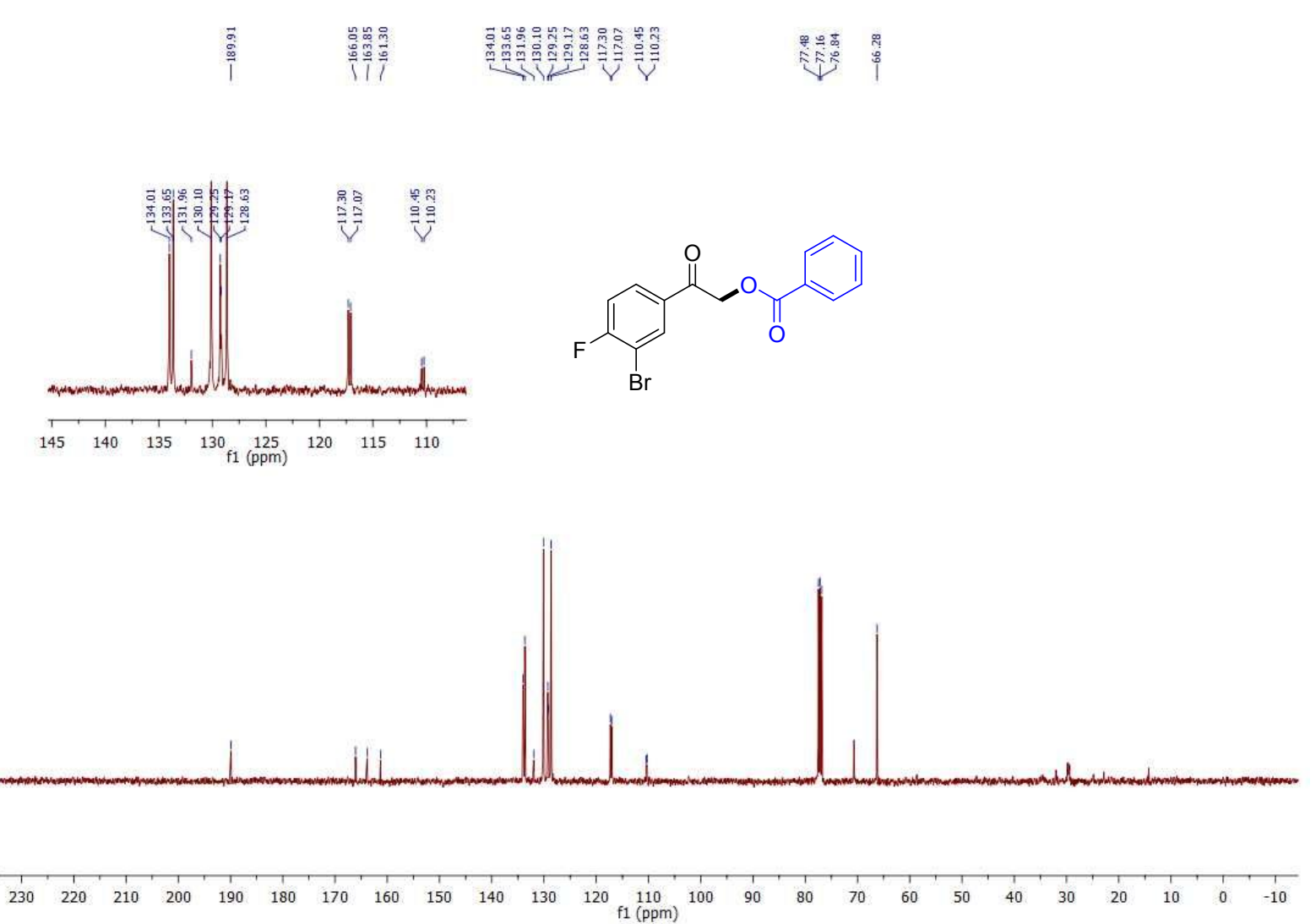

${ }^{13} \mathrm{C}$ NMR spectrum of $\mathbf{1 8}$ in $\mathrm{CDCl}_{3}$. 


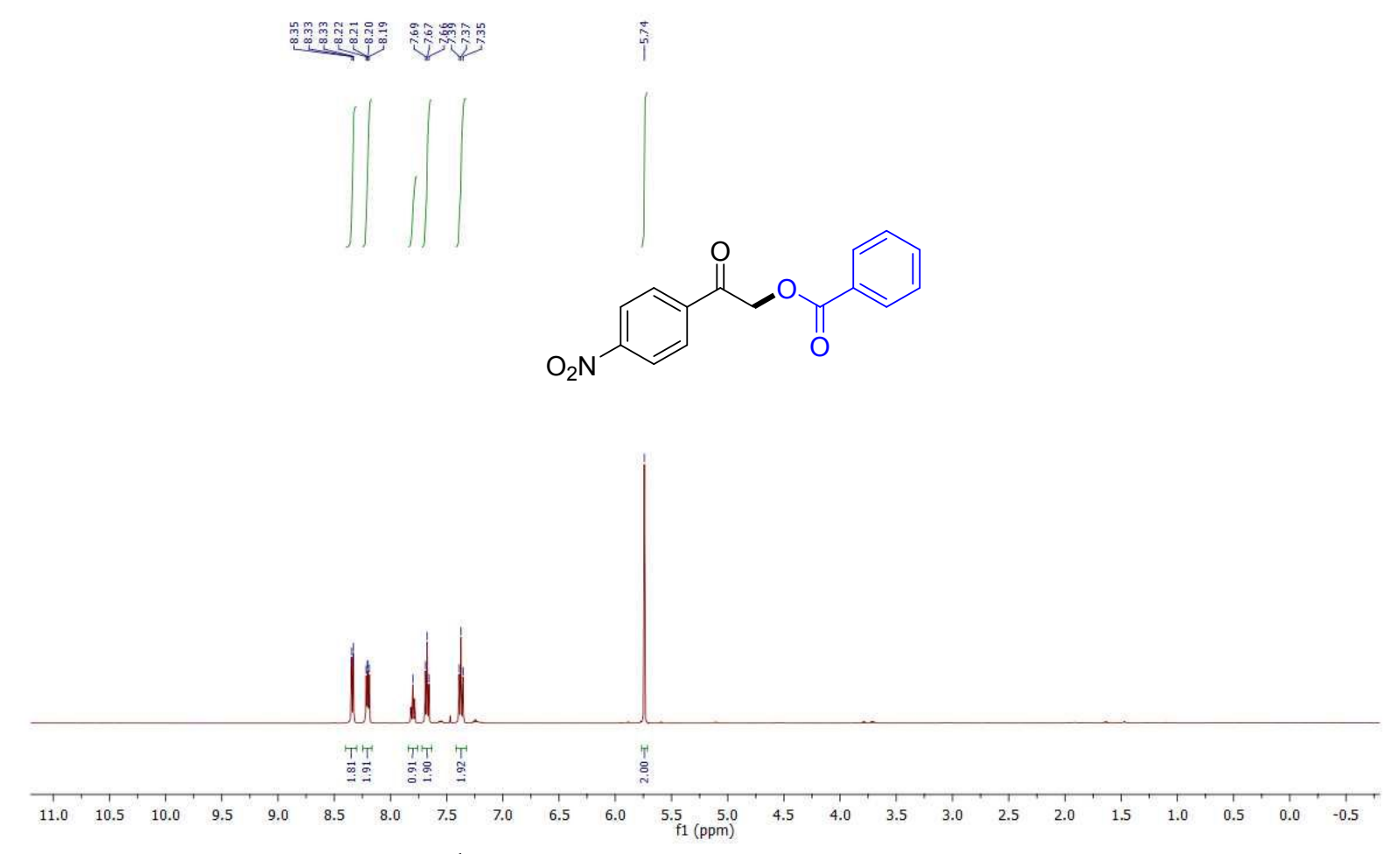

${ }^{1} \mathrm{H} \mathrm{NMR}$ spectrum of 19 in $\mathrm{CDCl}_{3}$.

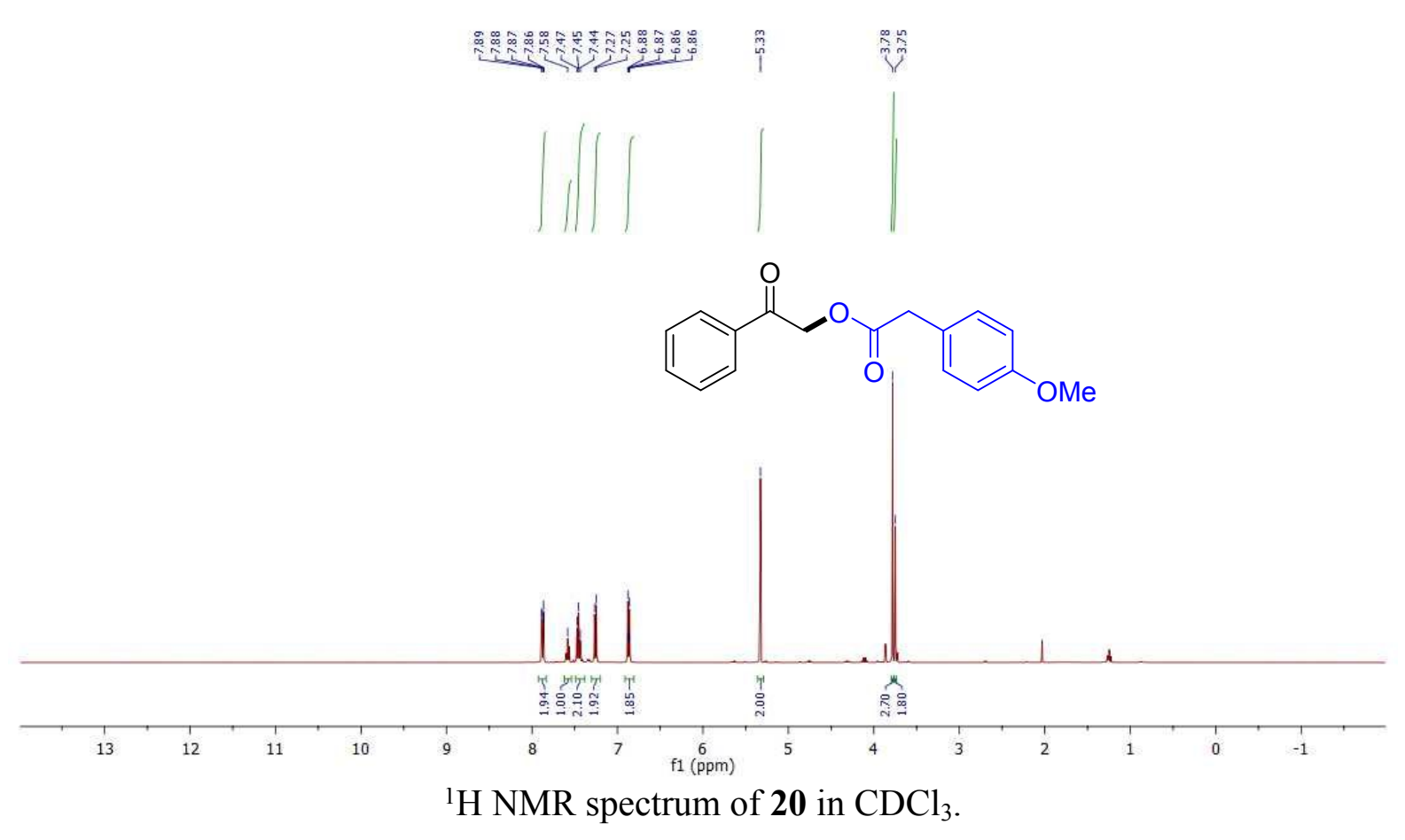




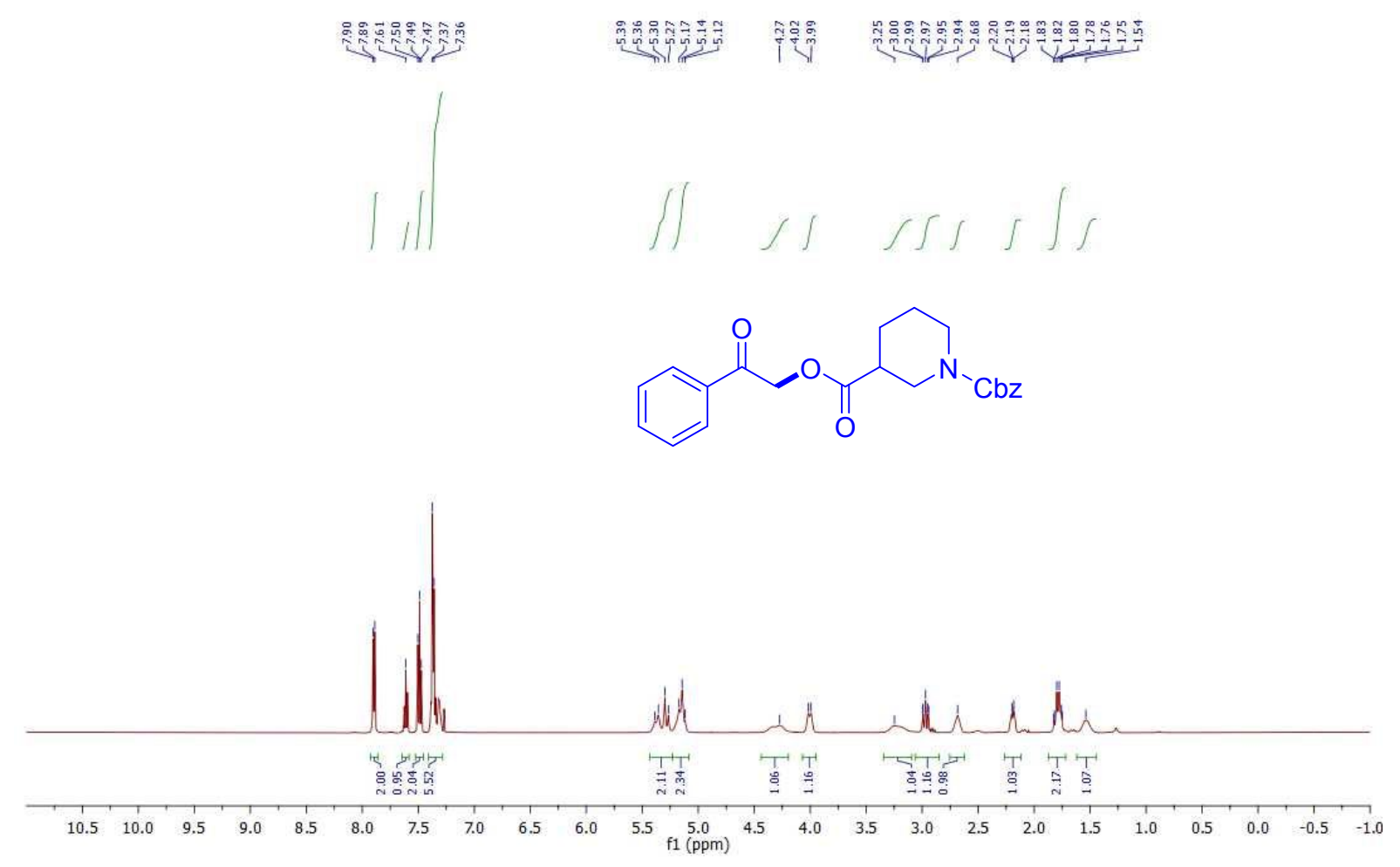

${ }^{1} \mathrm{H}$ NMR spectrum of 21 in $\mathrm{CDCl}_{3}$.

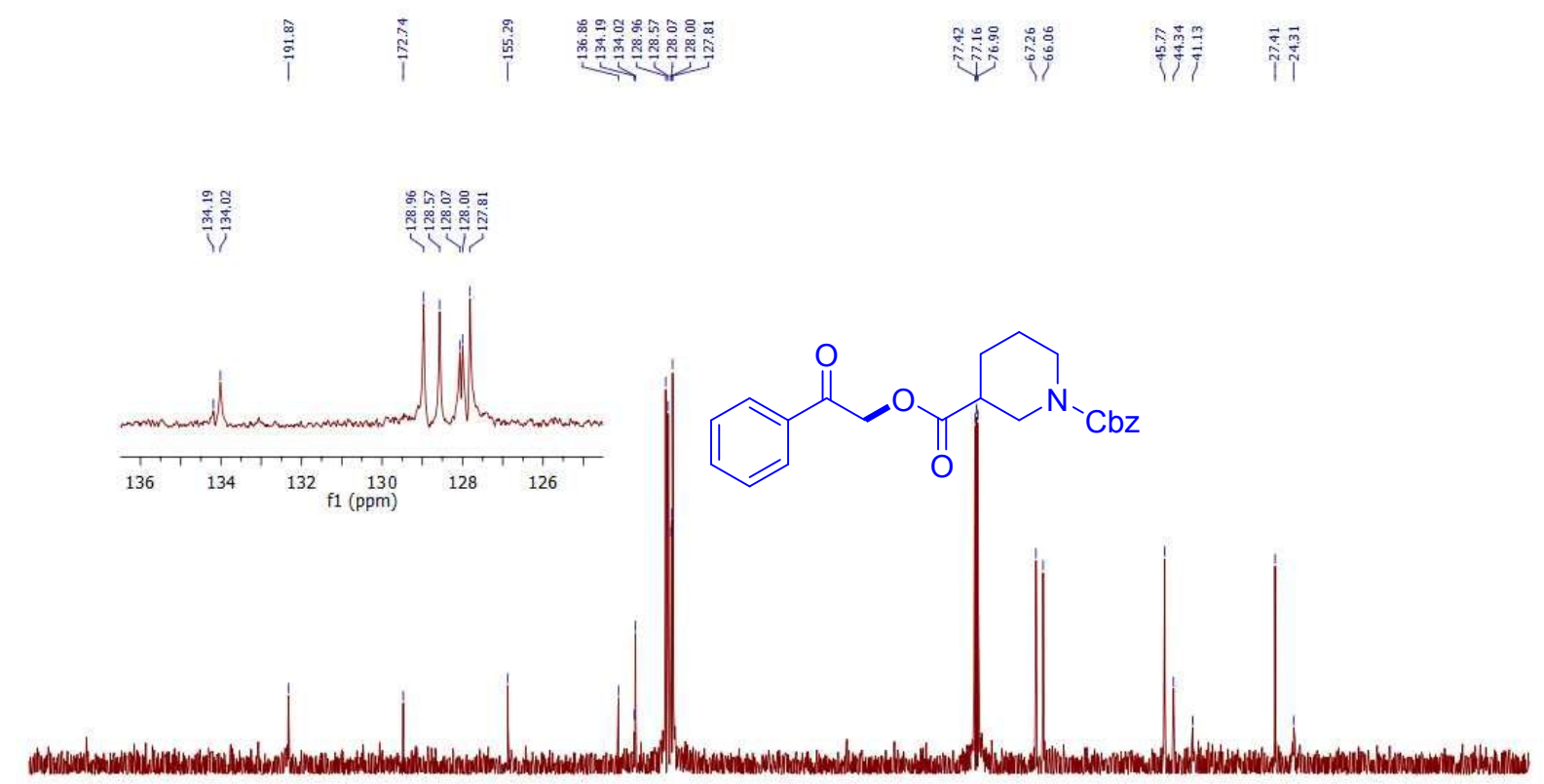

$\begin{array}{lllllllllllllllllllllllllllll}230 & 220 & 210 & 200 & 190 & 180 & 170 & 160 & 150 & 140 & 130 & 120 & 110 & 100 & 90 & 80 & 70 & 60 & 50 & 40 & 30 & 20 & 10 & 0 & -10\end{array}$

${ }^{13} \mathrm{C}$ NMR spectrum of 21 in $\mathrm{CDCl}_{3}$. 


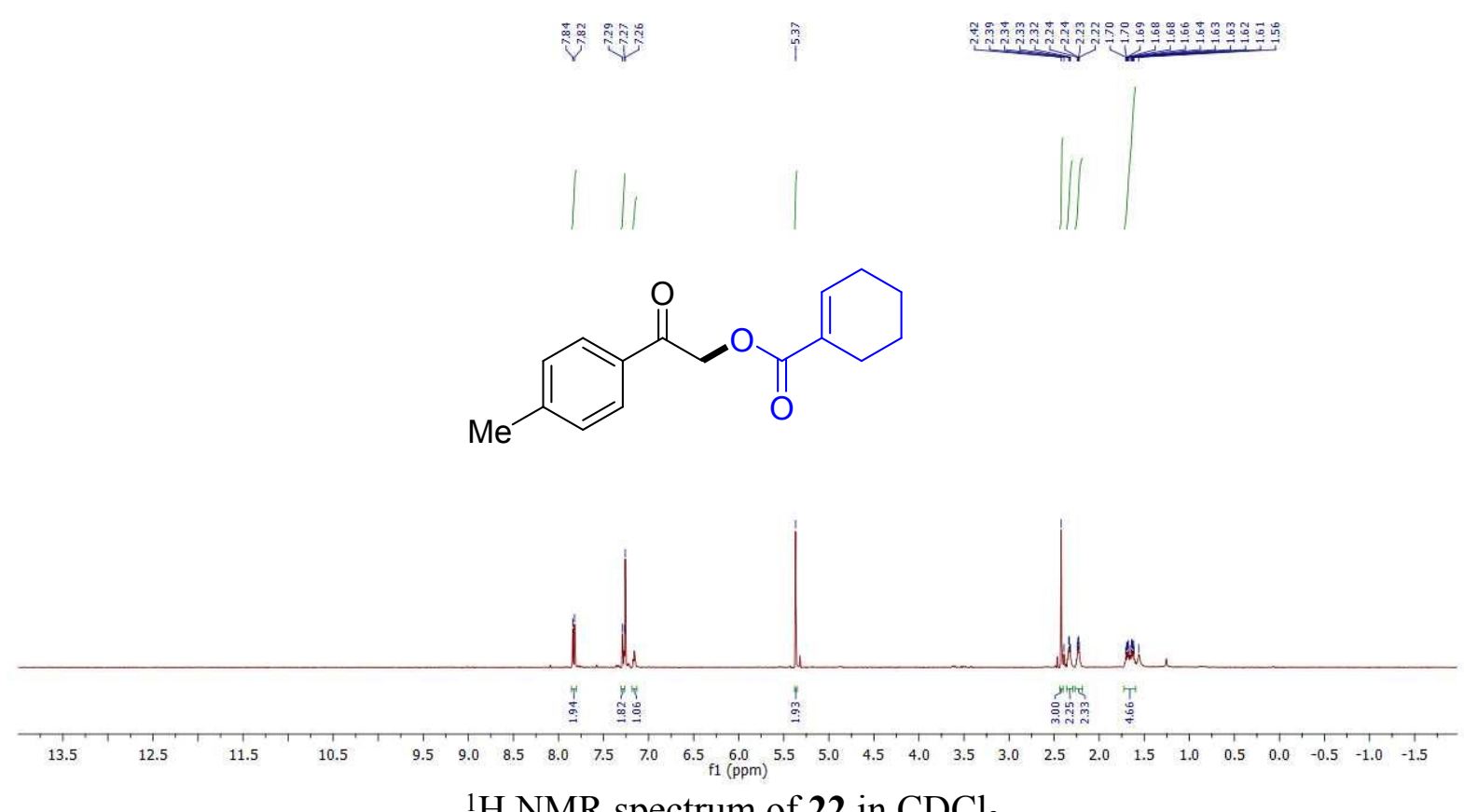

${ }^{1} \mathrm{H}$ NMR spectrum of 22 in $\mathrm{CDCl}_{3}$.

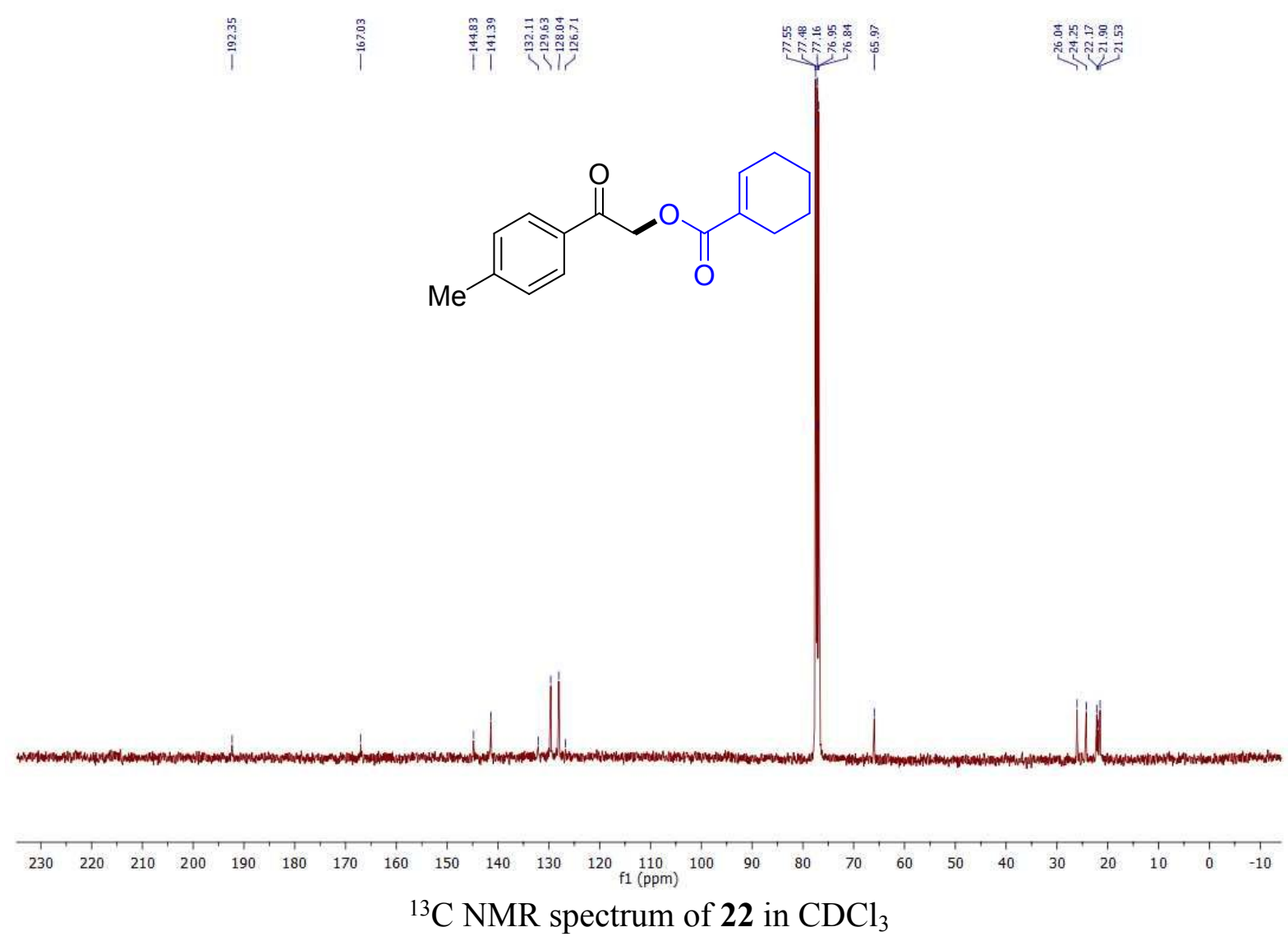




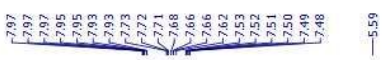
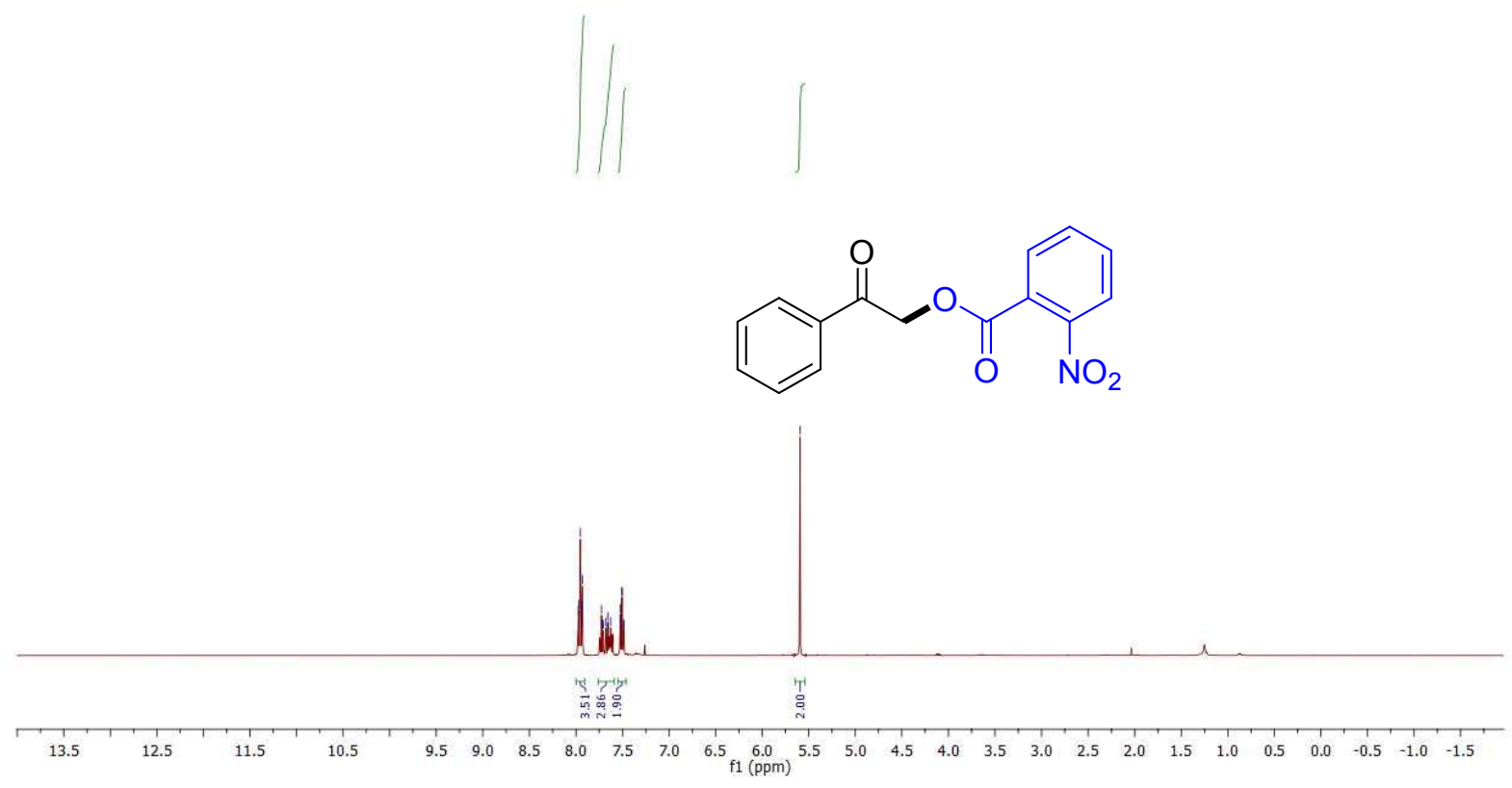

${ }^{1} \mathrm{H}$ NMR spectrum of 23 in $\mathrm{CDCl}_{3}$.
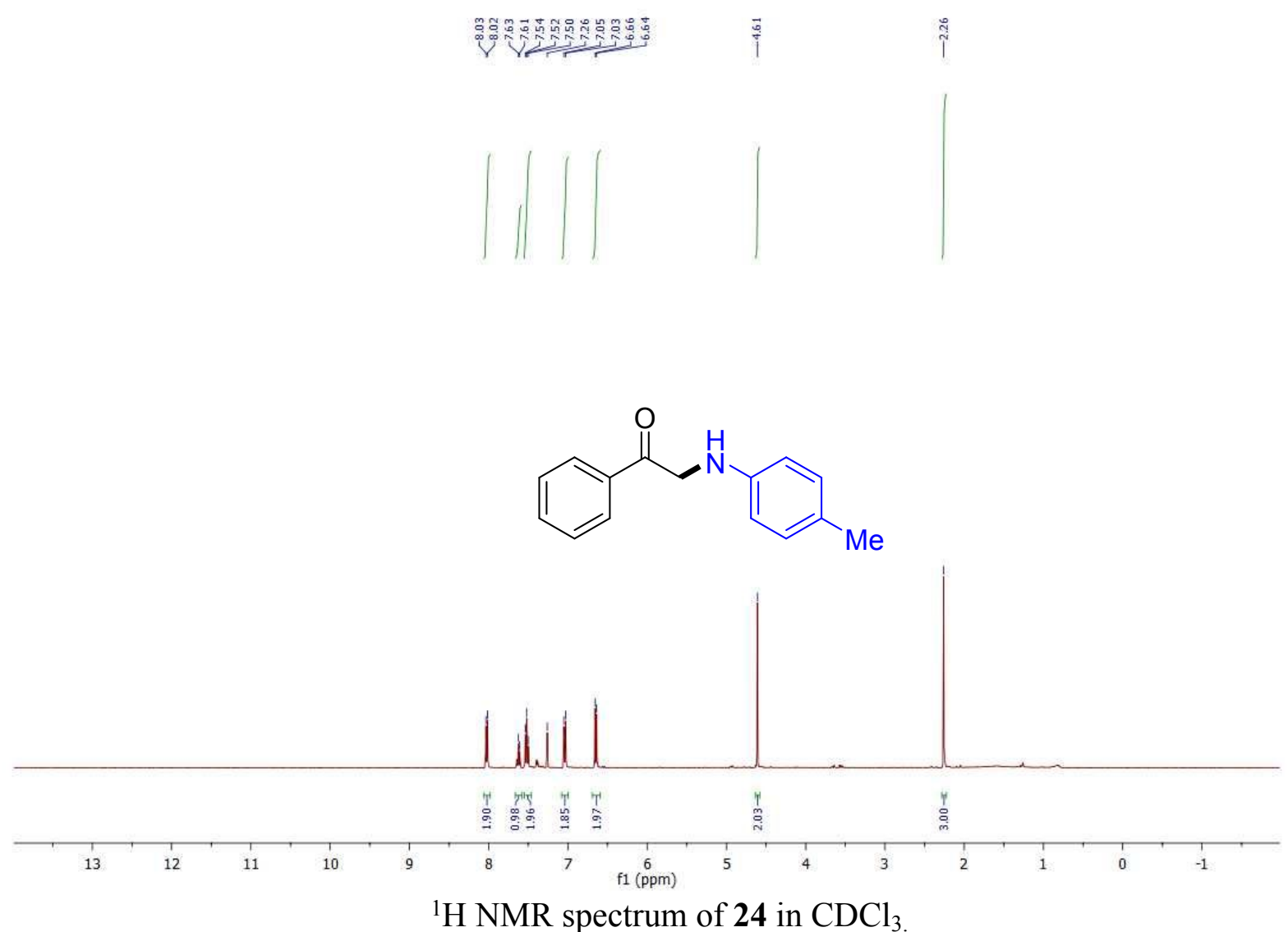

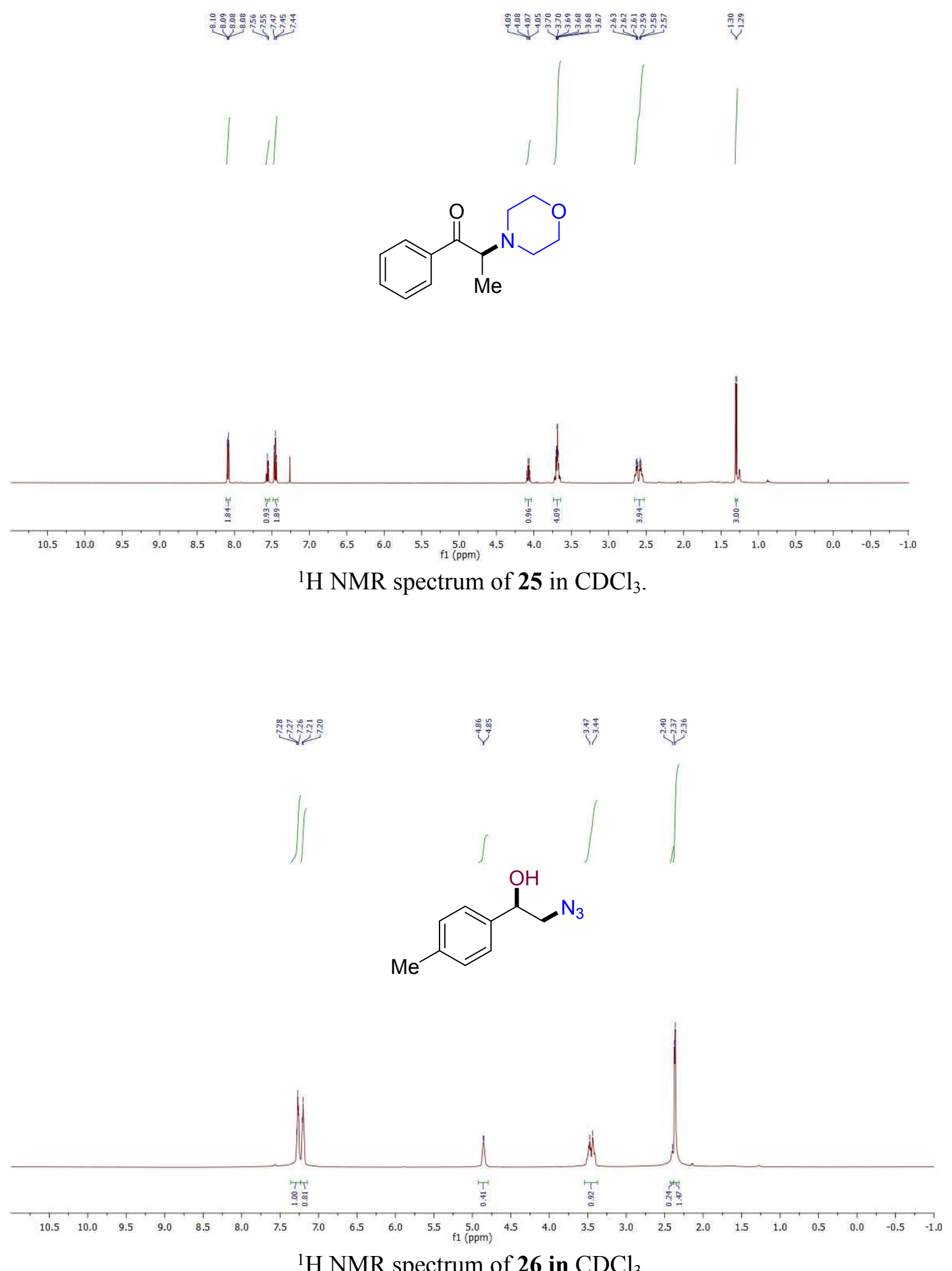

${ }^{1} \mathrm{H}$ NMR spectrum of 26 in $\mathrm{CDCl}_{3}$. 


\section{婹要路

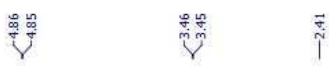

${ }^{1} \mathrm{H}$
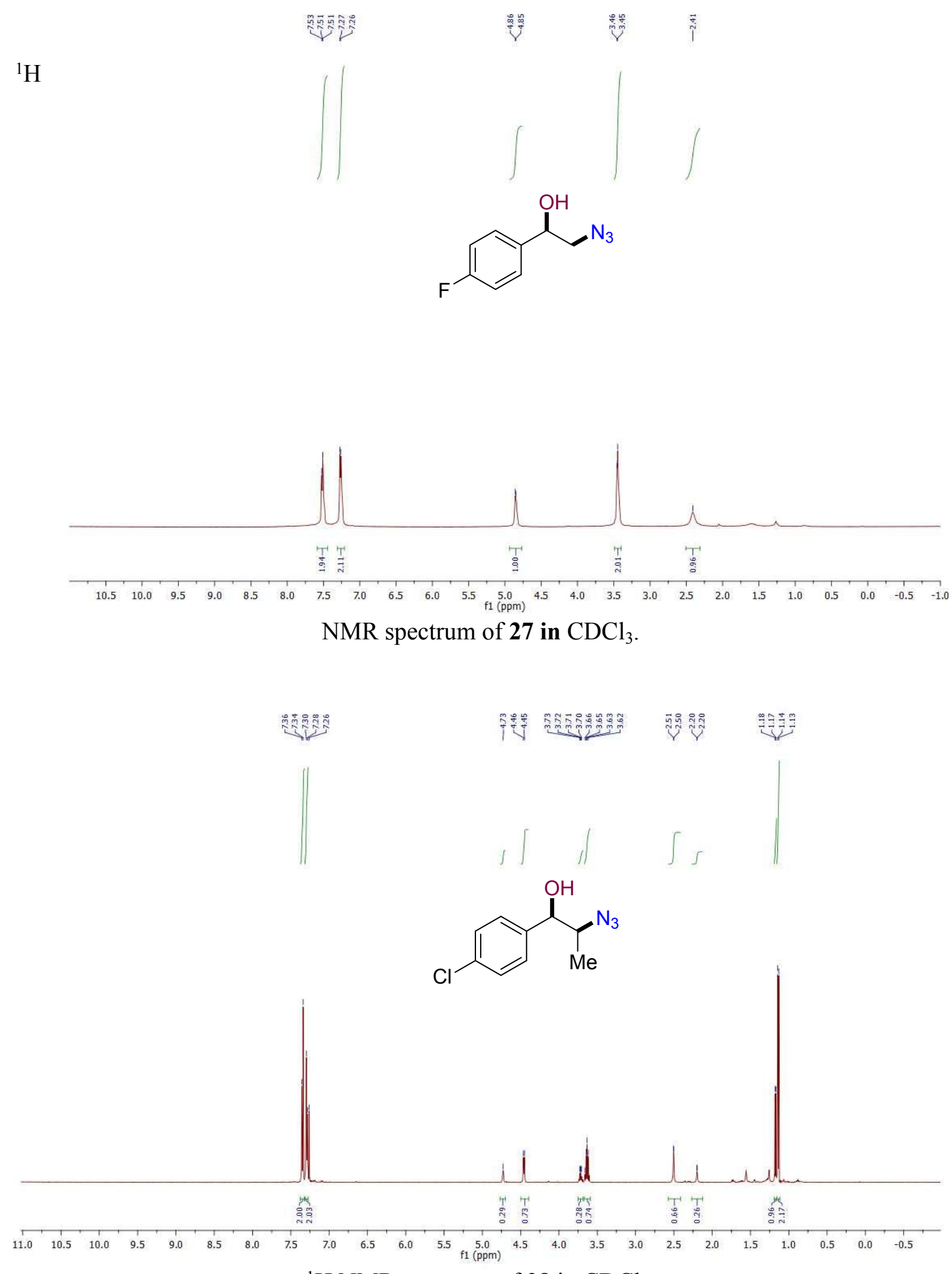

${ }^{1} \mathrm{H}$ NMR spectrum of 28 in $\mathrm{CDCl}_{3}$. 
<smiles>[N+]=[N+]([O-])C(O)C(O)c1ccc(Cl)cc1</smiles>

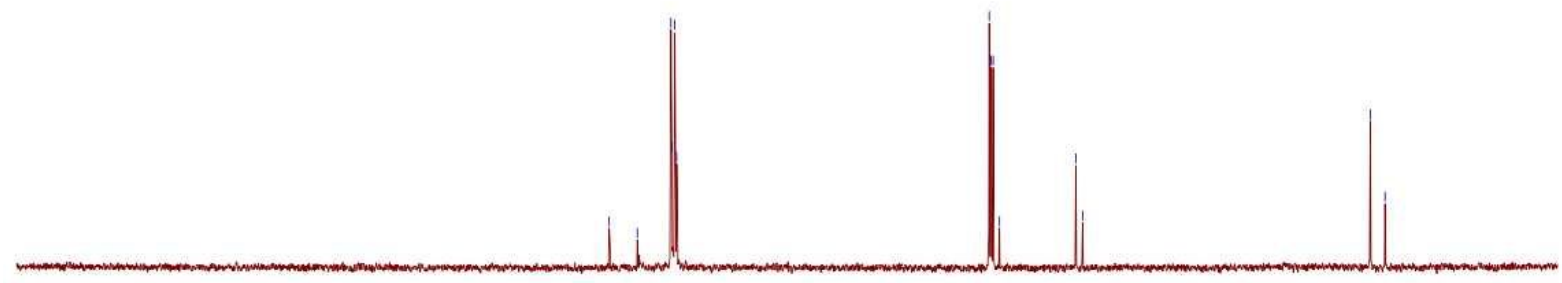
$\begin{array}{lllllllllllllllllllllllllllll}230 & 220 & 210 & 200 & 190 & 180 & 170 & 160 & 150 & 140 & 130 & 120 & 110 & 100 & 90 & 80 & 70 & 60 & 50 & 40 & 30 & 20 & 10 & 0 & -10\end{array}$

${ }^{13} \mathrm{C}$ NMR spectrum of 28 in $\mathrm{CDCl}_{3}$.

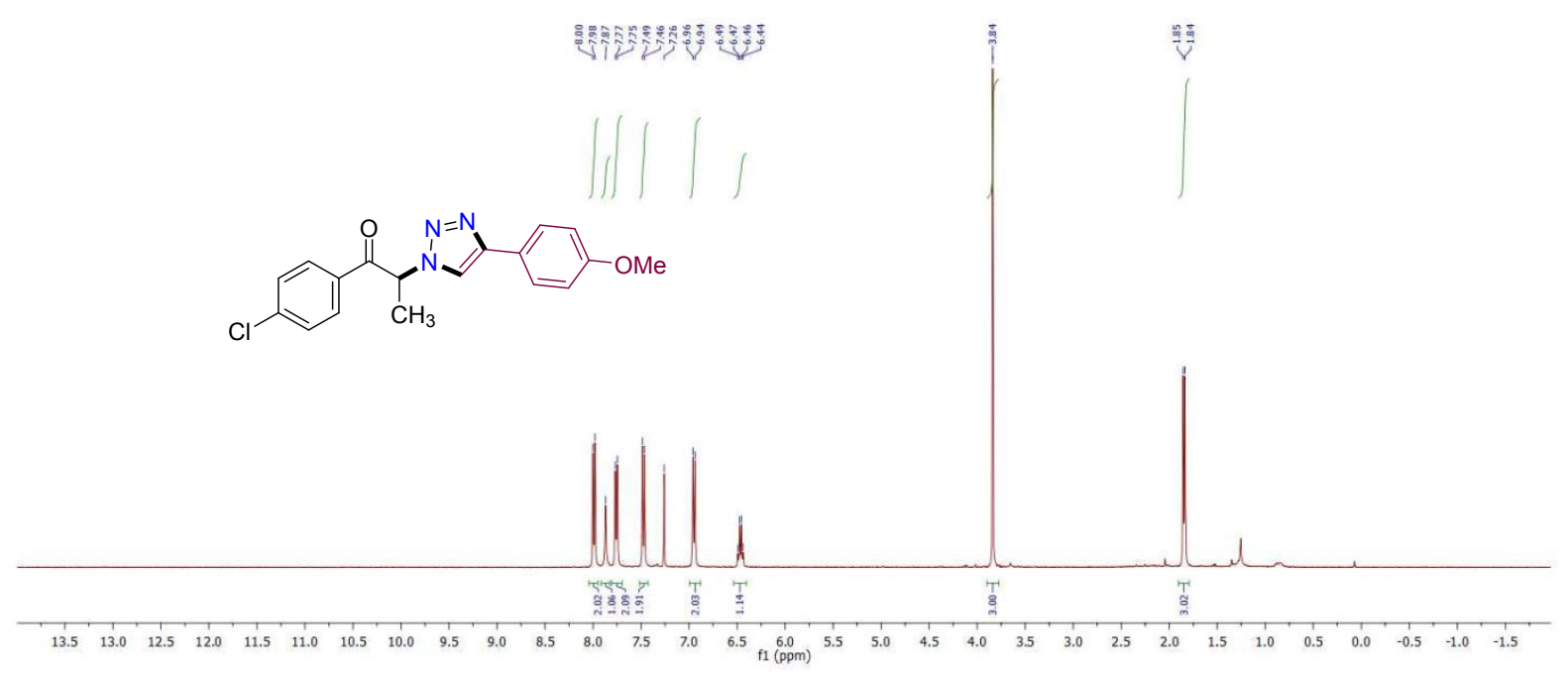

${ }^{1} \mathrm{H} \mathrm{NMR}$ spectrum of 29 in $\mathrm{CDCl}_{3}$. 


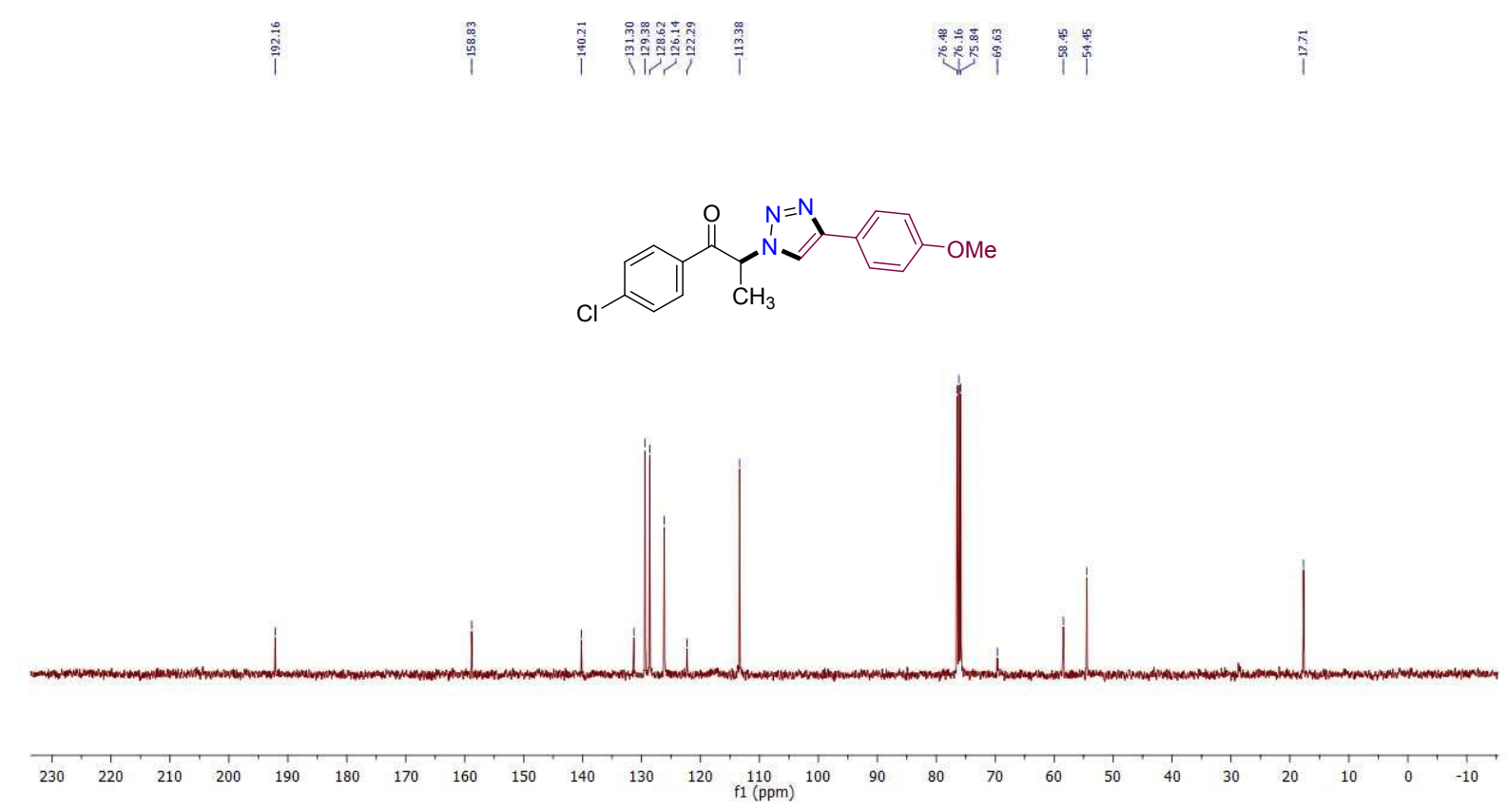

${ }^{13} \mathrm{C}$ NMR spectrum of 29 in $\mathrm{CDCl}_{3}$.

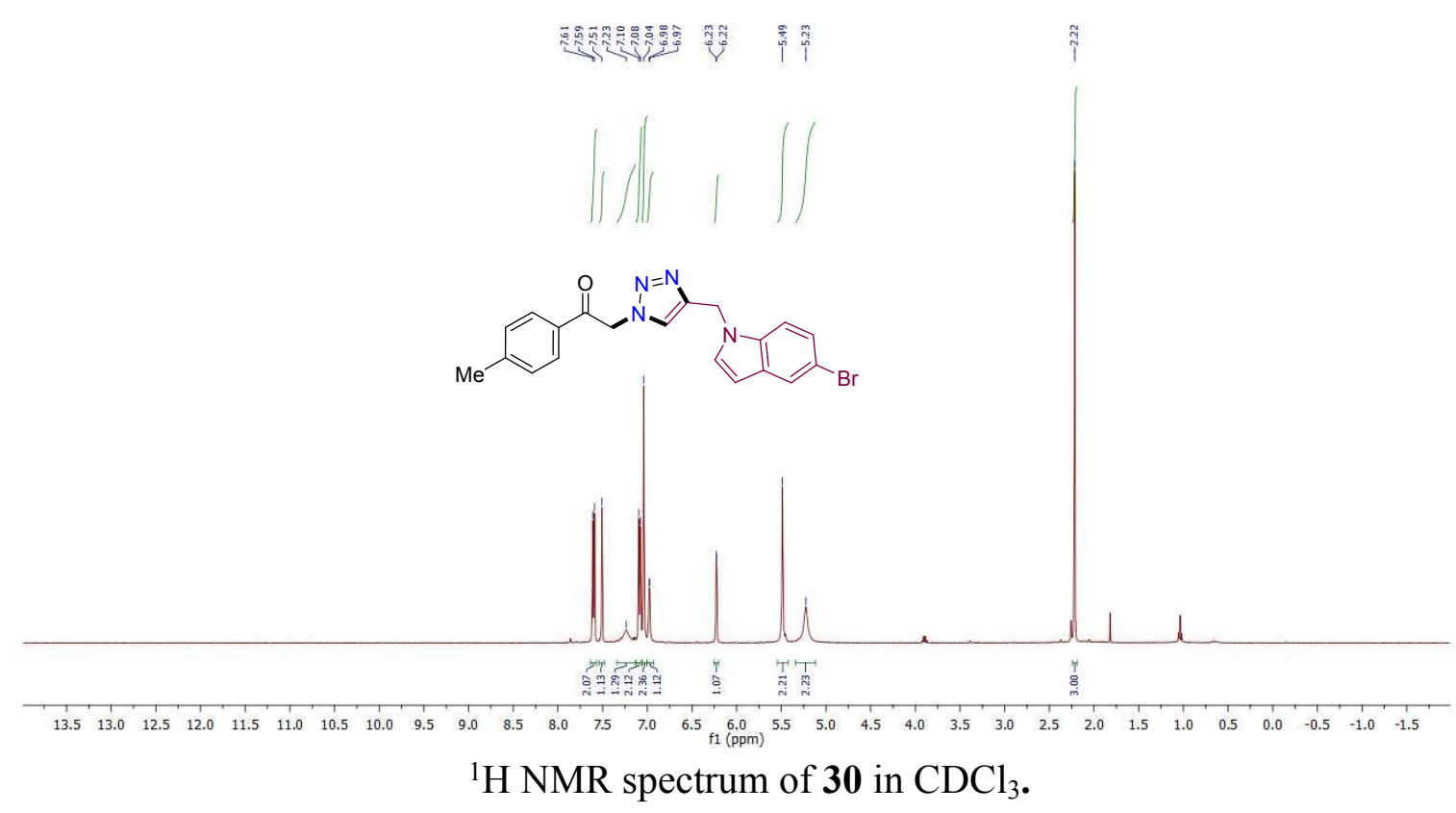




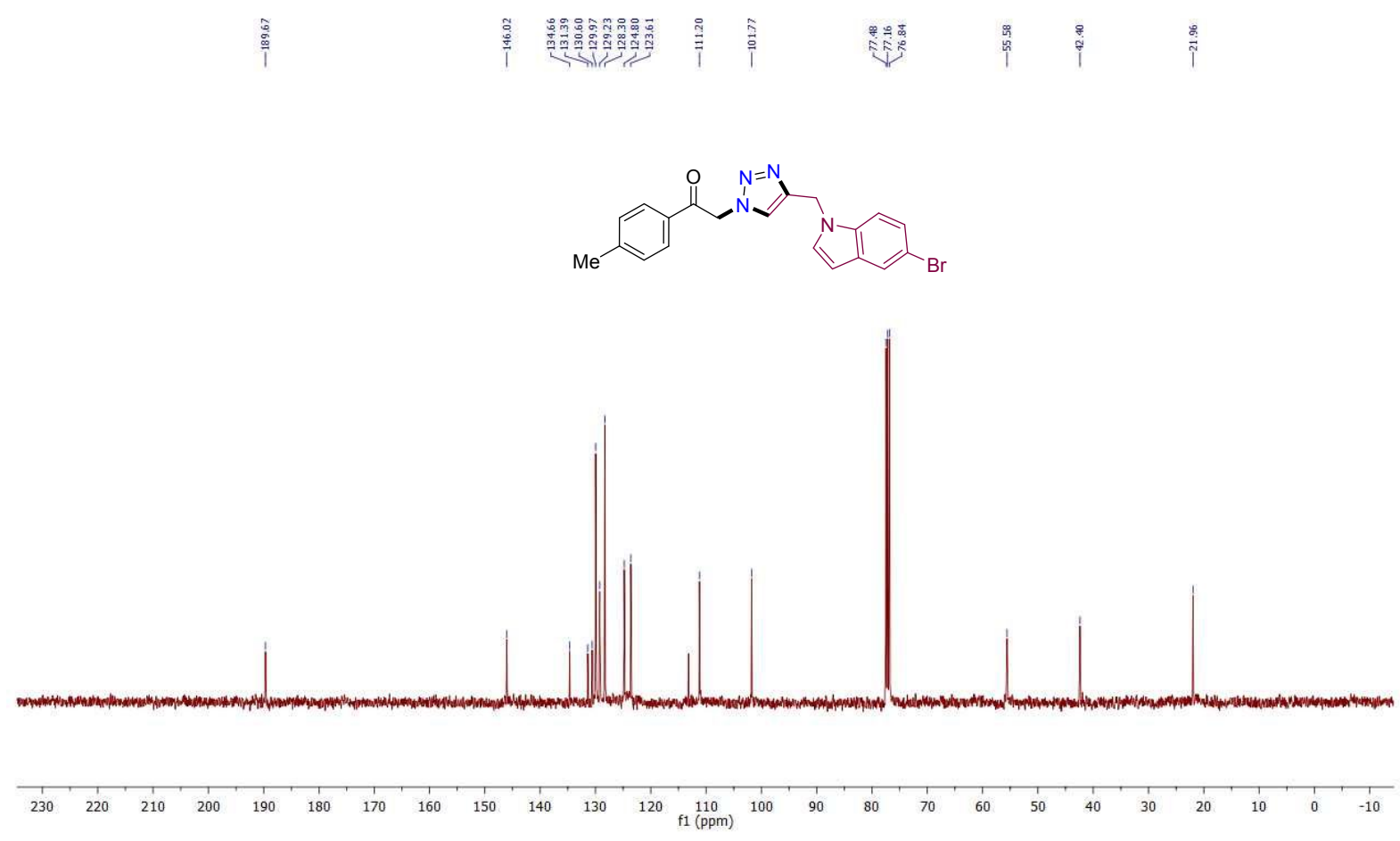

${ }^{13} \mathrm{C}$ NMR spectrum of $\mathbf{3 0}$ in $\mathrm{CDCl}_{3}$. 